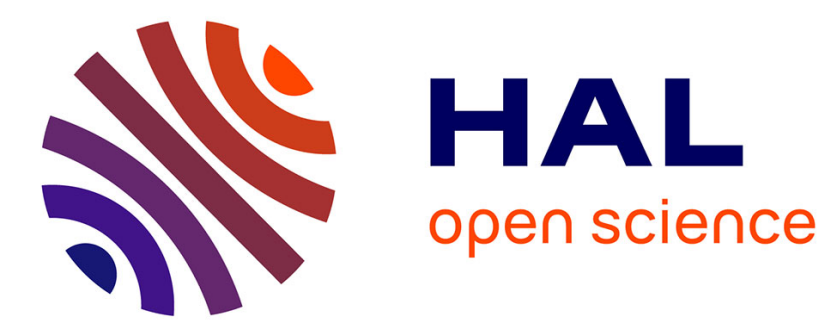

\title{
Vibratory control of a linear system by addition of a chain of nonlinear oscillators
}

Simon Charlemagne, Claude-Henri Lamarque, Alireza Ture Savadkoohi

\section{To cite this version:}

Simon Charlemagne, Claude-Henri Lamarque, Alireza Ture Savadkoohi. Vibratory control of a linear system by addition of a chain of nonlinear oscillators. Acta Mechanica, 2017, 228, pp.3111-3133. 10.1007/s00707-017-1867-7 . hal-01517957

\section{HAL Id: hal-01517957 \\ https://hal.science/hal-01517957}

Submitted on 21 Nov 2017

HAL is a multi-disciplinary open access archive for the deposit and dissemination of scientific research documents, whether they are published or not. The documents may come from teaching and research institutions in France or abroad, or from public or private research centers.
L'archive ouverte pluridisciplinaire HAL, est destinée au dépôt et à la diffusion de documents scientifiques de niveau recherche, publiés ou non, émanant des établissements d'enseignement et de recherche français ou étrangers, des laboratoires publics ou privés. 


\title{
S. Charlemagne · C.-H. Lamarque · A. Ture Savadkoohi
}

\section{Vibratory control of a linear system by addition of a chain of nonlinear oscillators}

\author{
S. Charlemagne $(\varangle) \cdot$ C.-H. Lamarque · A. T. Savadkoohi \\ ENTPE, LTDS UMR CNRS 5513, Univ. Lyon, Rue Maurice Audin, 69518 Vaulx-en-Velin Cedex, France \\ E-mail: simon.charlemagne@entpe.fr \\ C.-H. Lamarque \\ E-mail: claude.lamarque@entpe.fr \\ A. T. Savadkoohi \\ E-mail: alireza.turesavadkoohi@entpe.fr
}

\begin{abstract}
An $N$-degree-of-freedom model consisting of a single-degree-of-freedom linear system coupled to a chain of $(N-1)$ light nonlinear oscillators is studied. The connection between the chain and the singledegree-of-freedom system is supposed to be linear. Time multi-scale system behaviors at fast and slow time scales are investigated and lead to the detection of the slow invariant manifold and equilibrium and singular points. These points correspond to periodic regimes and strongly modulated responses, respectively. These analytical developments are used to provide evidence of transfer of vibratory energy of the main system to the chain in the form of localized modes during periodic regimes and extreme energy exchanges between modes when the overall structure faces singularities. Furthermore, analytical predictions at slow time scale and nonlinear normal modes of the system are compared with numerical results obtained from direct time integration of the system equations, showing a good agreement between them. Finally, we present a procedure showing how these analytical developments can be used to study a system where the main structure is replaced by a multi-degree-of-freedom linear system, by projecting its dynamics on one of its modes.
\end{abstract}

\section{Introduction}

Passive control of structural systems by addition of nonlinear devices has been extensively studied. Numerous works investigate the dynamics of two degree-of-freedom systems where a nonlinear oscillator called as nonlinear energy sink (NES) is used to localize vibratory energy of a main structure [1-4], among which some consider a linear main system and a NES with a cubic restoring force [5-9], leading to applications in various fields such as aerospace [10] or acoustical [11] engineering. Other types of nonlinearities of the NES have been studied as well, e.g., non-polynomial NES [12], vibro-impact NES [13] or piece-wise linear NES with timevarying mass [14], while some works focus on vibratory energy mitigation of nonlinear main systems such as hysteretic oscillators $[15,16]$. The aforementioned theoretical studies have been validated by experimental investigations. As an example, McFarland et al. [17] and Kerschen et al. [18] found experimental evidence of passive control of a linear structure performed by a cubic NES. In parallel, nonlinear oscillators have proven to be of interest in the control of multi-degree-of-freedom (MDOF) or continuous systems. They can engage either in sequential resonance captures of several modes of the primary system, known as resonance capture cascades 
$[19,20]$, or in relaxation oscillations, known as strongly modulated response (SMR) cycles when modes of the main system are closely spaced [21]. One can find other examples in the work of Gendelman and Manevitch [22], Vakakis et al. [23], Manevitch et al. [24], and Rothos and Vakakis [25] who study energy pumping achieved by a nonlinear attachment connected to semi-infinite continuous string and rod and semi-infinite and infinite linear chains of oscillators, respectively.

Nonetheless, it can be convenient for design reasons to couple multiple NES to the structure. It could for instance contribute to the robustness of the control in case one of the NES is damaged. Some studies considered several NES in parallel of the main system. Vaurigaud et al. [26] prove that a set of tuned parallel NES provides good performances in terms of energy transfer along with a better distribution of the mass. Experimental testings carried out by Ture Savadkoohi et al. [27] on a four degree-of-freedom structure coupled to two NES corroborate these conclusions. Meanwhile, some works investigated localization of energy of main systems in a set of three NES in series [28-30]. Those MDOF nonlinear attachments have been shown to be very efficient to absorb energy from a MDOF linear system under impulsive excitation. Indeed, those devices are able to dissipate energy from several modes simultaneously, involving a different mechanism as compared to single-degree-of-freedom (SDOF) NES. Similarly, Wierschem et al. [31] performed experimental testing on a six-story structure coupled to a NES made of two nonlinear oscillators in series. Finally, a comparable idea has been investigated by Starosvetsky and Vakakis [32] who study shock mitigation thanks to an $n$-beads granular interface placed between two rods.

In this paper, we aim to extend this idea to the study of a higher number of nonlinear light oscillators in series. A main linear system, subjected to external excitation, is coupled to a chain of $(N-1)$ nonlinear oscillators in order to mitigate its vibratory energy. This study presents a fully analytical method, based on complexification-averaging technique, to design a chain of nonlinear coupled oscillators as a passive controller and/or energy harvester of linear primary systems subjected to narrow-band harmonic excitation, thus involving a single mode of the main system under the assumption that all modes are well separated.

The present work is organized as follows. The studied system is presented in Sect. 2, along with the analytical method implemented for the treatment of the equations. System behaviors at fast and slow time scales are described in Sect. 3. Analytical predictions are compared to numerical simulations obtained thanks to direct time integration of the system equations in Sect. 4, while Sect. 5 aims to find evidence of passive control of the main linear structure. In Sect. 6, a procedure to apply the developed analytical method to the study of a system where the main SDOF linear structure is replaced by a MDOF linear system is presented. Finally, concluding remarks are given in Sect. 7.

\section{Description of the system and explanation of the analytical method}

The studied system is presented in Fig. 1. It consists of a linear SDOF structure with the mass $M$, the stiffness $K$, the damping $C$, under external excitation $F(t)$, which is linearly coupled to a chain of nonlinear oscillators via a damping $c$ and a linear spring $\rho$. The chain consists of $(N-1)$ nonlinear oscillators, each one has the mass $m=\epsilon M, 0<\epsilon \ll 1$. The connection between each of these oscillators is made up by a linear damping $c$ and a restoring force $V$ which contains linear and nonlinear contributions. The SDOF main system can result from the projection of an MDOF linear system on one of its normal modes which we would aim to control. This assumption will be verified in Sect. 6.

Displacements of the main system and the nonlinear oscillators are called as $v$ and $u_{j}, j=1, \ldots, N-1$, respectively. Let us define relative displacements $w_{j}$ as new variables of the system:

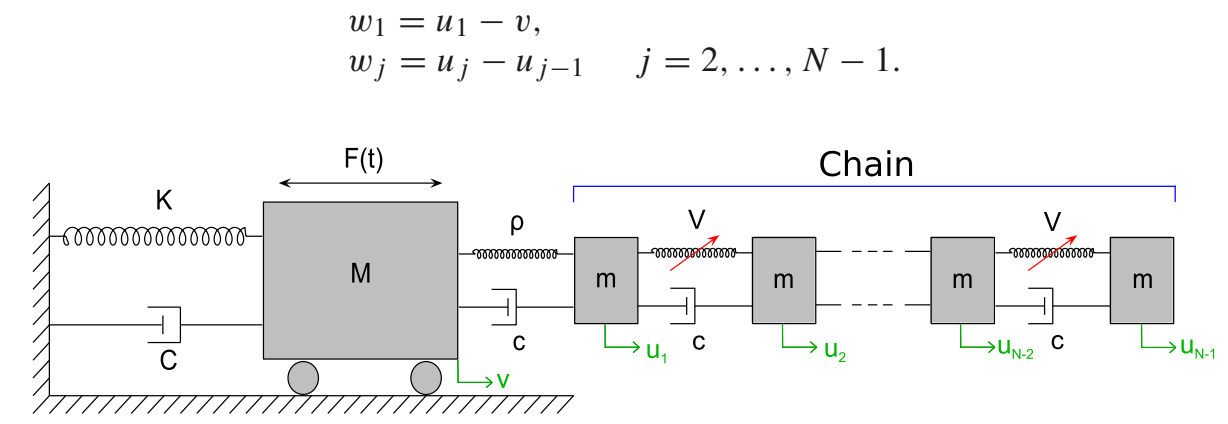

Fig. $1 N$-DOF model consisting of a forced linear structure coupled to $(N-1)$ nonlinear oscillators $(m=\epsilon M, 0<\epsilon \ll 1)$ 
Furthermore, the following rescaled parameters are introduced: $\frac{K}{M}=\omega_{0}^{2}, \frac{\rho}{M}=\epsilon r, \frac{V(z)}{M}=\epsilon\left(B z+D z^{3}\right)$, $\frac{C}{M}=\epsilon c, \frac{c}{M}=\epsilon c_{1}$, and $\frac{F(t)}{M}=\epsilon f^{0} \sin (\omega t)$. The linear stiffness $r$ is chosen different from $B$ because it has a direct impact on the energy transfer from the main structure to the chain. The nonlinear contribution of the restoring force $V$ in the chain is chosen to be cubic. Furthermore, we aim to investigate the dynamics of the system around resonance of the main structure, so we assume that $\omega$ is close to $\omega_{0}$, i.e., $\omega^{2}=\omega_{0}^{2}(1+\sigma \epsilon)$, where $\sigma$ is a detuning parameter. The governing equations of the system now read:

$$
\ddot{\mathbf{y}}+\left(\mathbf{C}_{0}+\epsilon \mathbf{C}_{1}\right) \dot{\mathbf{y}}+\left(\mathbf{B}_{0}+\epsilon \mathbf{B}_{1}\right) \mathbf{y}+D \mathcal{N}(\mathbf{y})=\epsilon \mathcal{F}(t)
$$

where

$$
\begin{aligned}
& \mathbf{B}_{0}=\left(\begin{array}{ccccccccc}
\omega_{0}^{2} & 0 & 0 & \ldots & \ldots & \cdots & \cdots & \ldots & 0 \\
-\omega_{0}^{2} & r & -B & \ddots & & & & & \vdots \\
0 & -r & 2 B & -B & \ddots & & & & \vdots \\
\vdots & \ddots & -B & 2 B & -B & \ddots & & & \vdots \\
\vdots & & \ddots & -B & 2 B & -B & \ddots & & \vdots \\
\vdots & & & \ddots & \ddots & \ddots & \ddots & \ddots & \vdots \\
\vdots & & & & \ddots & \ddots & \ddots & \ddots & 0 \\
\vdots & & & & & \ddots & \ddots & \ddots & -B \\
0 & \ldots & \ldots & \ldots & \ldots & \ldots & 0 & -B & 2 B
\end{array}\right), \\
& \mathbf{C}_{0}=\left(\begin{array}{cccccc}
0 & 0 & 0 & \ldots & \ldots & 0 \\
0 & c_{1} & -c_{1} & \ddots & & \vdots \\
0 & -c_{1} & 2 c_{1} & -c_{1} & \ddots & \vdots \\
\vdots & \ddots & \ddots & \ddots & \ddots & 0 \\
\vdots & & \ddots & -c_{1} & 2 c_{1} & -c_{1} \\
0 & \ldots & \ldots & 0 & -c_{1} & 2 c_{1}
\end{array}\right) \\
& \mathbf{B}_{1}=\left(\begin{array}{ccccc}
0 & -r & 0 & \ldots & 0 \\
0 & r & 0 & \ldots & 0 \\
0 & 0 & 0 & \ldots & 0 \\
\vdots & \vdots & \vdots & \ddots & \vdots \\
0 & 0 & 0 & \ldots & 0
\end{array}\right), \quad \mathbf{C}_{1}=\left(\begin{array}{ccccc}
c & -c_{1} & 0 & \ldots & 0 \\
-c & c_{1} & 0 & \ldots & 0 \\
0 & 0 & 0 & \ldots & 0 \\
\vdots & \vdots & \vdots & \ddots & 0 \\
0 & 0 & 0 & \ldots & 0
\end{array}\right), \\
& \mathcal{N}(\mathbf{y})=\left(\begin{array}{c}
0 \\
-w_{2}^{3} \\
2 w_{2}^{3}-w_{3}^{3} \\
-w_{2}^{3}+2 w_{3}^{3}-w_{4}^{3} \\
\vdots \\
-w_{j-1}^{3}+2 w_{j}^{3}-w_{j+1}^{3} \\
\vdots \\
-w_{N-3}^{3}+2 w_{N-2}^{3}-w_{N-1}^{3} \\
-w_{N-2}^{3}+2 w_{N-1}^{3}
\end{array}\right), \quad \mathbf{y}=\left(\begin{array}{c}
v \\
w_{1} \\
\vdots \\
w_{N-1}
\end{array}\right), \quad \mathcal{F}(t)=\left(\begin{array}{c}
f^{0} \sin (\omega t) \\
-f^{0} \sin (\omega t) \\
0 \\
\vdots \\
0
\end{array}\right)
\end{aligned}
$$

As $\mathbf{B}_{0}$ is diagonalizable, there exists a matrix $\mathbf{P}_{0}$ such that $\mathbf{P}_{0}^{-1} \mathbf{B}_{0} \mathbf{P}_{0}=\boldsymbol{\Lambda}_{0}^{2}$, i.e., $\mathbf{B}_{0}$ and $\boldsymbol{\Lambda}_{0}^{2}$ are similar matrices. Furthermore, we define $\tilde{\boldsymbol{\Lambda}}_{0}$ as $\tilde{\boldsymbol{\Lambda}}_{0}^{2}=\boldsymbol{\Lambda}_{0}^{2}-\omega^{2} \mathbf{I} \mathbf{d}_{N}$, where $\mathbf{I d}_{N}$ is the $N \times N$ identity matrix. System (2.1) can 
be rewritten as:

$$
\ddot{\mathbf{y}}+\omega^{2} \mathbf{y}+\left(\mathbf{C}_{0}+\epsilon \mathbf{C}_{1}\right) \dot{\mathbf{y}}+\mathbf{P}_{0} \tilde{\mathbf{\Lambda}}_{0}^{2} \mathbf{P}_{0}^{-1} \mathbf{y}+\epsilon \mathbf{B}_{1} \mathbf{y}+\mathcal{N}(\mathbf{y})=\epsilon \mathcal{F}(t)
$$

To treat this system of $N$ nonlinear equations, the method described in [33] is used, keeping the equations in a discrete form. Three analytical tools are implemented:

- A time multiple scales method [34] is used: time is embedded to fast $\tau_{0}=t$ and slow $\tau_{k}=\epsilon^{k} t, k=1,2, \ldots$ time scales. The system equations will be derived at different orders of $\epsilon$ in order to study the dynamics at corresponding time scales.

- Complex variables of Manevitch [35] are introduced to the system (4):

$$
\begin{gathered}
\boldsymbol{\Phi} e^{i \omega t}=\dot{\mathbf{y}}+i \omega \mathbf{y} \\
\text { with } \boldsymbol{\Phi}=\left(\begin{array}{c}
\varphi_{1} \\
\vdots \\
\varphi_{N}
\end{array}\right) \text { and } i=\sqrt{-1}
\end{gathered}
$$

- A Galerkin technique is carried out in order to obtain a Fourier series truncated at first harmonic. For a generic function $s\left(\tau_{1}, \tau_{2}, \ldots\right)$, it reads:

$$
S=\frac{\omega}{2 \pi} \int_{0}^{\frac{2 \pi}{\omega}} s\left(\tau_{1}, \tau_{2}, \ldots\right) e^{-i \omega \tau_{0}} d \tau_{0} .
$$

To apply (6), we suppose that $\boldsymbol{\Phi}$ is independent of $\tau_{0}$, which will be eventually verified at least for an asymptotic state, i.e., at slow time scales. We also keep $\dot{\boldsymbol{\Phi}}$ in the equations.

Finally, system (4) reads:

$$
\begin{gathered}
\dot{\boldsymbol{\Phi}}+\frac{1}{2}\left(\mathbf{C}_{0}+\epsilon \mathbf{C}_{1}\right) \boldsymbol{\Phi}+\frac{1}{2 i \omega}\left(\mathbf{P}_{0} \tilde{\mathbf{\Lambda}}_{0}^{2} \mathbf{P}_{0}^{-1}+\epsilon \mathbf{B}_{1}\right) \boldsymbol{\Phi}-\frac{3 i D}{8 \omega^{3}} \mathcal{N}_{m}(\boldsymbol{\Phi})=\epsilon \mathcal{F}_{m}(t), \\
0 \\
-\left|\varphi_{3}\right|^{2} \varphi_{3} \\
2\left|\varphi_{3}\right|^{2} \varphi_{3}-\left|\varphi_{4}\right|^{2} \varphi_{4} \\
-\left|\varphi_{3}\right|^{2} \varphi_{3}+2\left|\varphi_{4}\right|^{2} \varphi_{4}-\left|\varphi_{5}\right|^{2} \varphi_{5} \\
\vdots \\
\vdots \\
-\left|\varphi_{j-1}\right|^{2} \varphi_{j-1}+2\left|\varphi_{j}\right|^{2} \varphi_{j}-\left|\varphi_{j+1}\right|^{2} \varphi_{j+1} \\
\vdots \\
-\left|\varphi_{N-2}\right|^{2} \varphi_{N-2}+2\left|\varphi_{N-1}\right|^{2} \varphi_{N-1}-\left|\varphi_{N}\right|^{2} \varphi_{N} \\
-\left|\varphi_{N-1}\right|^{2} \varphi_{N-1}+2\left|\varphi_{N}\right|^{2} \varphi_{N} \\
\left(\begin{array}{c}
f^{0} \\
2 i \\
-\frac{f^{0}}{2 i} \\
0 \\
\vdots \\
0
\end{array}\right) \\
\mathcal{F}_{m}(t)=
\end{gathered}
$$




\section{Characterization of the system at different time scales}

\subsection{System behavior at fast time scale $\tau_{0}$}

The system behavior at fast time scale is described by the system (7) derived at the $\epsilon^{0}$ order:

$$
\begin{gathered}
\frac{\partial \boldsymbol{\Phi}}{\partial \tau_{0}}+\frac{1}{2} \mathbf{C}_{0} \boldsymbol{\Phi}+\frac{1}{2 i \omega_{0}} \mathbf{P}_{0} \tilde{\mathbf{\Lambda}}_{0}^{2} \mathbf{P}_{0}^{-1} \boldsymbol{\Phi}-i \mathcal{D} \mathcal{N}_{m}(\boldsymbol{\Phi})=0 \\
\Leftrightarrow \frac{\partial \boldsymbol{\Phi}}{\partial \tau_{0}}+\left(\begin{array}{c}
0 \\
\mathcal{H}_{2}(\boldsymbol{\Phi}) \\
\vdots \\
\mathcal{H}_{N}(\boldsymbol{\Phi})
\end{array}\right)=0
\end{gathered}
$$

where $\mathcal{D}=\frac{3 D}{8 \omega_{0}^{3}}$. Thus, $\varphi_{1}$ is independent of $\tau_{0}$ time scale.

The fixed points of system (9) verify $\lim _{\tau_{0} \rightarrow+\infty} \frac{\partial \tilde{\boldsymbol{\Phi}}}{\partial \tau_{0}}=0$ where $\tilde{\boldsymbol{\Phi}}=\left(\phi_{1} \ldots \phi_{N}\right)^{T}$. They define the slow invariant manifold (SIM) of the system:

$$
\left\{\begin{aligned}
\mathcal{H}_{2}= & \frac{i \omega_{0}}{2} \phi_{1}+\left(\frac{i \omega_{0}}{2}+\frac{c_{1}}{2}-\frac{i r}{2 \omega_{0}}\right) \phi_{2}+\alpha \phi_{3}+i \mathcal{D}\left|\phi_{3}\right|^{2} \phi_{3}=0 \\
\mathcal{H}_{3}= & \left(-\frac{c_{1}}{2}+\frac{i r}{2 \omega_{0}}\right) \phi_{2}+\frac{i \omega_{0}}{2} \phi_{3}+\alpha\left(-2 \phi_{3}+\phi_{4}\right) \\
& +i \mathcal{D}\left(-2\left|\phi_{3}\right|^{2} \phi_{3}+\left|\phi_{4}\right|^{2} \phi_{4}\right)=0 \\
\vdots & \\
\mathcal{H}_{j}= & \alpha\left(\phi_{j-1}-2 \phi_{j}+\phi_{j+1}\right)+\frac{i \omega_{0}}{2} \phi_{j} \\
& +i \mathcal{D}\left(\left|\phi_{j-1}\right|^{2} \phi_{j-1}-2\left|\phi_{j}\right|^{2} \phi_{j}+\left|\phi_{j+1}\right|^{2} \phi_{j+1}\right)=0 \\
& j=4, \ldots, N-1, \\
& \vdots \\
\mathcal{H}_{N}= & \alpha\left(\phi_{N-1}-2 \phi_{N}\right)+\frac{i \omega_{0}}{2} \phi_{N}+i \mathcal{D}\left(\left|\phi_{N-1}\right|^{2} \phi_{N-1}-2\left|\phi_{N}\right|^{2} \phi_{N}\right)=0
\end{aligned}\right.
$$

where $\alpha=-\frac{c_{1}}{2}+\frac{i B}{2 \omega_{0}}$.

Equation (10) consists of $(N-1)$ complex equations with $N$ complex unknown variables. Introducing polar coordinates $\phi_{j}=N_{j} e^{i \delta_{j}}, j=1, \ldots, N$, expressions of magnitudes of $(N-1)$ variables $\phi_{j}$ along with all phase differences can be derived with respect to the magnitude of the remaining variable. For example, for a given value of $N_{N}$, values of $N_{j}, j=1, \ldots, N-1$ as well as $\Delta_{j}=\delta_{j}-\delta_{j-1}, j=2, \ldots, N$ can be computed. If the value of one phase $\delta_{j}$ is set, then all phases can be calculated as well. To summarize, the SIM collects all possible asymptotic regimes of the system. However, one needs another complex equation in addition to the SIM to determine completely the system, i.e., to calculate the two remaining unknown variables (we will assume that these two real variables are $N_{N}$ and $\delta_{1}$ ). This extra information is given in Sect. 3.2 with the study at slow time scale $\tau_{1}$.

Stability of the SIM can be traced by an infinitesimal linear perturbation of $\boldsymbol{\Phi}$ in system (9) around the SIM as:

$$
\left(\begin{array}{c}
\varphi_{2} \\
\vdots \\
\varphi_{N}
\end{array}\right) \rightarrow\left(\begin{array}{c}
\phi_{2} \\
\vdots \\
\phi_{N}
\end{array}\right)+\Delta \tilde{\boldsymbol{\Phi}}, \Delta \tilde{\boldsymbol{\Phi}}=\left(\begin{array}{c}
\Delta \phi_{2} \\
\vdots \\
\Delta \phi_{N}
\end{array}\right)
$$


As $\varphi_{1}$ is independent of $\tau_{0}$, it is not perturbed. Linearizing the obtained system by keeping only the first-order terms in $\boldsymbol{\Delta} \tilde{\boldsymbol{\Phi}}$ leads to the following matrix system:

$$
\left(\begin{array}{c}
\frac{\partial \Delta \tilde{\boldsymbol{\Phi}}}{\partial \tau_{0}} \\
\frac{\partial \boldsymbol{\Delta} \tilde{\boldsymbol{\Phi}}^{*}}{\partial \tau_{0}}
\end{array}\right)=\boldsymbol{\Sigma}\left(\begin{array}{c}
\boldsymbol{\Delta} \tilde{\boldsymbol{\Phi}} \\
\boldsymbol{\Delta} \tilde{\boldsymbol{\Phi}}^{*}
\end{array}\right)
$$

where.$^{*}$ stands for the complex conjugate and $\boldsymbol{\Sigma}$ is a $(2 N-2) \times(2 N-2)$ matrix. Points where at least one eigenvalue of $\boldsymbol{\Sigma}$ has a positive real part determine unstable zones of the SIM.

\subsection{System behavior at slow time scale $\tau_{1}$}

Let us consider first the equation of system (7) derived at the $\epsilon^{1}$ order:

$$
\frac{\partial \varphi_{1}}{\partial \tau_{1}}+\frac{1}{2}\left(i \sigma \omega_{0}+c\right) \varphi_{1}+\frac{1}{2}\left(\frac{i r}{\omega_{0}}-c_{1}\right) \varphi_{2}+\frac{i f^{0}}{2}=0 .
$$

This equation provides necessary additional information to detect equilibrium and singular points at slow time scale around the SIM. Equilibrium points predict periodic regimes of the system: In this case, each mass oscillates at a given constant amplitude determined by the values of $N_{j}$ and $\delta_{j}(j=1, \ldots, N)$ on the equilibrium point. Singular points can lead to strongly modulated response (SMR) [36] which is characterized by persistent bifurcations of the system around its unstable zones. Equation (13) and the SIM read:

$$
\begin{gathered}
\frac{\partial \phi_{1}}{\partial \tau_{1}}+\left(\frac{i \sigma \omega_{0}}{2}+\frac{c}{2}\right) \phi_{1}+\left(\frac{i r}{2 \omega_{0}}-\frac{c_{1}}{2}\right) \phi_{2}+\frac{i f^{0}}{2}=0, \\
\Leftrightarrow\left\{\begin{array}{l}
\frac{\partial N_{1}}{\partial \tau_{1}}=-\frac{c}{2} N_{1}+\left(\frac{r}{2 \omega_{0}} \sin \left(\Delta_{2}\right)+\frac{c_{1}}{2} \cos \left(\Delta_{2}\right)\right) N_{2}-\frac{f^{0}}{2} \sin \left(\delta_{1}\right)=E_{1} \\
\frac{\partial \delta_{1}}{\partial \tau_{1}}=-\frac{\sigma \omega_{0}}{2}-\left(\frac{r}{2 \omega_{0}} \cos \left(\Delta_{2}\right)-\frac{c_{1}}{2} \sin \left(\Delta_{2}\right)\right) \frac{N_{2}}{N_{1}}-\frac{f^{0}}{2 N_{1}} \cos \left(\delta_{1}\right)=E_{2} .
\end{array}\right.
\end{gathered}
$$

As equilibrium and singular points are sought on the SIM, variables $N_{1}, N_{2}$, and $\Delta_{2}$ are replaced in system (14) by their expression obtained from Eq. (10). Moreover, one can obtain from the equations of the SIM:

$$
\begin{gathered}
\left\{\begin{array}{l}
\frac{\partial \mathcal{H}_{j, r}}{\partial \tau_{1}}=0 \\
\frac{\partial \mathcal{H}_{j, i}}{\partial \tau_{1}}=0
\end{array} \quad j=2, \ldots, N,\right. \\
\Leftrightarrow \mathbf{S}_{2}\left(\begin{array}{c}
\frac{\partial N_{2}}{\partial \tau_{1}} \\
\vdots \\
\frac{\partial N_{N}}{\partial \tau_{1}} \\
\frac{\partial \delta_{2}}{\partial \tau_{1}} \\
\vdots \\
\frac{\partial \delta_{N}}{\partial \tau_{1}}
\end{array}\right)=-\mathbf{S}_{1}\left(\begin{array}{c}
\frac{\partial N_{1}}{\partial \tau_{1}} \\
\frac{\partial \delta_{1}}{\partial \tau_{1}}
\end{array}\right)
\end{gathered}
$$


where $\mathcal{H}_{j, r}$ and $\mathcal{H}_{j, i}$ are real and imaginary parts of $\mathcal{H}_{j}$, respectively, and:

$$
\begin{aligned}
& \mathbf{S}_{1}=\frac{\omega_{0}}{2}\left(\begin{array}{cc}
-\sin \left(\delta_{1}\right) & -N_{1} \cos \left(\delta_{1}\right) \\
\cos \left(\delta_{1}\right) & -N_{1} \sin \left(\delta_{1}\right) \\
0 & 0 \\
\vdots & \vdots \\
0 & 0
\end{array}\right), \\
& \mathbf{S}_{2}=\left(\begin{array}{cccccc}
\frac{\partial \mathcal{H}_{2, r}}{\partial N_{2}} & \ldots & \frac{\partial \mathcal{H}_{2, r}}{\partial N_{N}} & \frac{\partial \mathcal{H}_{2, r}}{\partial \delta_{2}} & \ldots & \frac{\partial \mathcal{H}_{2, r}}{\partial \delta_{N}} \\
\frac{\partial \mathcal{H}_{2, i}}{\partial N_{2}} & \ldots & \frac{\partial \mathcal{H}_{2, i}}{\partial N_{N}} & \frac{\partial \mathcal{H}_{2, i}}{\partial \delta_{2}} & \ldots & \frac{\partial \mathcal{H}_{2, i}}{\partial \delta_{N}} \\
\vdots & & \vdots & \vdots & & \vdots \\
\frac{\partial \mathcal{H}_{N, r}}{\partial N_{2}} & \ldots & \frac{\partial \mathcal{H}_{N, r}}{\partial N_{N}} & \frac{\partial \mathcal{H}_{N, r}}{\partial \delta_{2}} & \ldots & \frac{\partial \mathcal{H}_{N, r}}{\partial \delta_{N}} \\
\frac{\partial \mathcal{H}_{N, i}}{\partial N_{2}} & \ldots & \frac{\partial \mathcal{H}_{N, i}}{\partial N_{N}} & \frac{\partial \mathcal{H}_{N, i}}{\partial \delta_{2}} & \ldots & \frac{\partial \mathcal{H}_{N, i}}{\partial \delta_{N}}
\end{array}\right) .
\end{aligned}
$$

Multiplying by the adjugate matrix of $\mathbf{S}_{2}$, i.e., $\operatorname{adj}\left(\mathbf{S}_{2}\right)$, and replacing $\frac{\partial N_{1}}{\partial \tau_{1}}$ and $\frac{\partial \delta_{1}}{\partial \tau_{1}}$ by their expressions obtained from system (14), system (15) reads:

$$
\operatorname{det}\left(\mathbf{S}_{2}\right) \mathbf{I}_{2 N-2}\left(\begin{array}{c}
\frac{\partial N_{2}}{\partial \tau_{1}} \\
\vdots \\
\frac{\partial N_{N}}{\partial \tau_{1}} \\
\frac{\partial \delta_{2}}{\partial \tau_{1}} \\
\vdots \\
\frac{\partial \delta_{N}}{\partial \tau_{1}}
\end{array}\right)=\underbrace{-\operatorname{adj}\left(\mathbf{S}_{2}\right) \mathbf{S}_{1}\left(\begin{array}{c}
E_{1} \\
E_{2}
\end{array}\right)}_{\mathcal{G}}
$$

where $\mathbf{I d}_{2 N-2}$ is the $(2 N-2) \times(2 N-2)$ unit matrix and $\mathcal{G}$ is a $(2 N-2)$ column vector. System (17) enables to detect both equilibrium and singular points. Equilibrium points verify [33]:

$$
\left\{\begin{array} { l } 
{ \operatorname { d e t } ( \mathbf { S } _ { 2 } ) \neq 0 } \\
{ E _ { 1 } = E _ { 2 } = 0 }
\end{array} \Leftrightarrow \left\{\begin{array}{l}
\operatorname{det}\left(\mathbf{S}_{2}\right) \neq 0 \\
\mathcal{G}=0
\end{array}\right.\right.
$$

Singular points verify:

$$
\left\{\begin{array}{l}
\operatorname{det}\left(\mathbf{S}_{2}\right)=0 \\
\mathcal{G}=0
\end{array}\right.
$$

\section{Comparison of numerical results to analytical predictions}

In this Section, numerical examples are confronted with analytical predictions. All numerical results are obtained via direct integration on the system equations (2.1). A Runge-Kutta scheme has been used thanks to the Matlab ${ }^{\circledR}$ function ode45. A 50-DOF system $(N=50$, the main structure is coupled to 49 nonlinear oscillators) is studied in this Section using the following parameters: $\epsilon=0.001, \omega_{0}=1, r=5, c=0.2$, $B=2, c_{1}=4$, and $D=50$. The forcing amplitude $f^{0}$ and the detuning parameter $\sigma$ will vary to describe different behaviors of the system. 


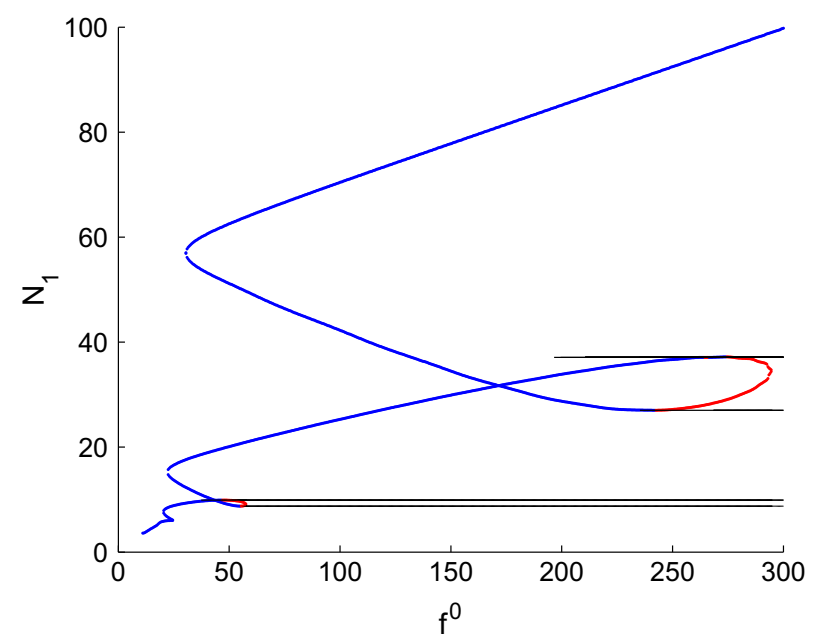

Fig. $2 N=50, \epsilon=0.001, \omega_{0}=1, r=5, c=0.2, B=2, c_{1}=4, D=50$, and $\sigma=0$ - $N_{1}$-amplitude of equilibrium and singular points depending on the forcing amplitude $f^{0}$. Stable and unstable equilibrium points are plotted in blue and red, respectively, while the presence of singular points is denoted by black solid lines (colour figure online)

Table $1 N_{1}$-amplitude of equilibrium points for three different forcing amplitudes and for the following parameters: $N=50$, $\epsilon=0.001, \omega_{0}=1, r=5, c=0.2, B=2, c_{1}=4, D=50$, and $\sigma=0$

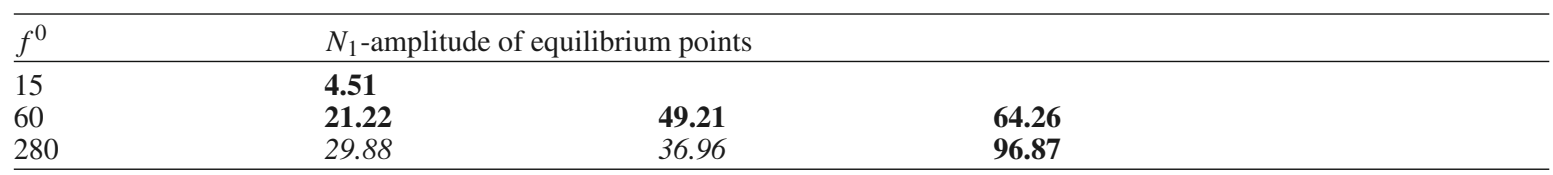

Values in bold and italic shapes relate to stable and unstable equilibrium points, respectively

\subsection{Periodic regimes}

We first aim to highlight the system behavior around equilibrium points, i.e., periodic regimes. As $f^{0}$ varies, the amplitude of equilibrium points changes, and singular points appear. Figure 2 depicts $N_{1}$-amplitude of equilibrium and singular points as a function of the forcing amplitude. It reveals the coexistence of multiple solutions for a wide interval of $f^{0}$ and the existence of unstable equilibrium points, i.e., points located on unstable branches of the SIM.

In this Subsection, three different values of forcing amplitude $f^{0}$ are chosen to describe three periodic regimes of the system. These values and the corresponding $N_{1}$-amplitudes of equilibrium points are given in Table 1.

Figure 3 shows, for the first case $f^{0}=15$, the SIM and the position of the equilibrium point (no. 1 ) of the system projected in the plane $\left(N_{50}, N_{1}\right)$. Starting from given initial conditions, $\mathbf{y}(t=0)=(60,0, \ldots, 0)$, the system should oscillate around the SIM and finally stabilize its behavior around the equilibrium point at low energy. Evolution of $N_{1}$ versus time taken from numerical results is plotted in Fig. 4a. It shows that, after a transient state, the system reaches equilibrium point no. 1. Comparison between numerical results at $\tau_{1}$ time scale and analytical predictions is provided in Fig. 4b. Blue circles denote the numerical amplitude of oscillation of each mass of the system at $\tau_{1}$ time scale, while red lines represent amplitudes of oscillation computed from equilibrium point no. 1. A qualitative agreement is verified since the general geometry of both profiles is similar. Besides, the prediction of the amplitude of the main system is quantitatively good as the relative error is about 3\%. Figure $4 \mathrm{c}$ plots the evolution of the amplitudes of oscillation of each mass of the chain (from the second to the last oscillator) on the equilibrium point no. 1. Analytical developments thus predict that energy will propagate through the chain along nonlinear characteristic curves. Corresponding numerical results given in Fig. 4d show a similar behavior.

For the second example, the system with $f^{0}=60$ possesses three equilibrium points (no. 1, 2, and 3) and two singular points located at the edge of the first unstable branch of the SIM as shown in Fig. 5. This implies that the system could either undergo SMR or stabilize its behavior around one of the three equilibrium points. As shown in Fig. $6 a-b$, starting from initial conditions $\mathbf{y}=(20,0, \ldots, 0)$, the numerical behavior tends to 


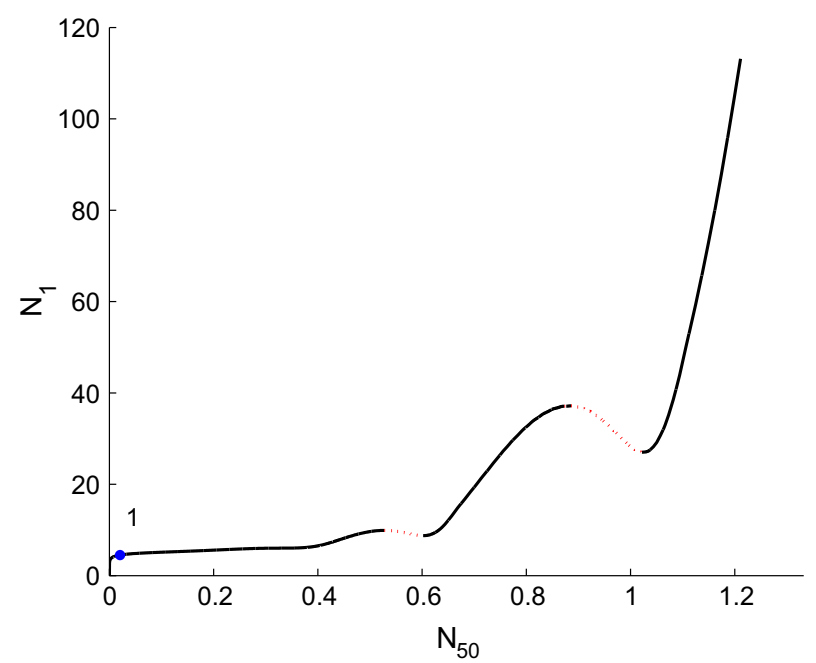

Fig. $3 N=50, \epsilon=0.001, \omega_{0}=1, r=5, c=0.2, B=2, c_{1}=4, D=50, \sigma=0$ and $f^{0}=15$-SIM of the system (black line) and position of the equilibrium point (blue point). Red dotted lines denote unstable branches of the SIM. The system possesses one equilibrium point (no. 1) (colour figure online)

equilibrium point no. 1. Besides, the analytical method gives a precise prediction of the asymptotic dynamics of the system. Such agreement is observed in Fig. $6 \mathrm{~b}$ as well as in the comparison of Fig. 6c with Fig. 6d. They both show the same modal behavior of the chain.

For the third example, the system with $f^{0}=280$ has three equilibrium points: nos. 1 and 2 are unstable, while no. 3 is stable. It also possesses six singular points at the boundaries of the three unstable branches of the SIM (see Fig. 7). Note that the second unstable branch is hard to detect as it is encased between the two abscissas $N_{50}=0.873$ and $N_{50}=0.8765$. Figure 8 shows that the system behavior tends to equilibrium point no. 3. Initial conditions are $\mathbf{y}=(60,0, \ldots, 0)$. As in the previous case, analytical and numerical results are in good agreement. The behavior illustrated at high energy shows that the chain is split in two groups of masses oscillating in an opposite phase relationship, separated by a minimum of amplitude of vibration located around the middle of the chain.

N.B. One should keep in mind that all parameters have been derived at orders of $\epsilon$, implying that $f^{0}$ (as well as other parameters) must be at $\epsilon^{0}$ order. As $\epsilon=0.001$ and $f^{0}=280$, the current example is a borderline case.

\subsection{Nonlinear normal modes}

Figures 6 and 8 of the previous Subsection depict a vibration in unison of the system. Therefore, it seems legitimate to wonder if the behaviors illustrated around equilibrium points are related to nonlinear normal modes of the system, defined as synchronous periodic motion of the underlying unforced Hamiltonian system [37-39]. As a result, we search periodic solutions of the form:

$$
\mathbf{y}=\mathbf{Y} \cos (\Omega t), \mathbf{Y}=\left(\begin{array}{c}
V \\
W_{1} \\
\vdots \\
W_{N-1}
\end{array}\right)=\left(\begin{array}{c}
V \\
U_{1}-V \\
\vdots \\
U_{N-1}-U_{N-2}
\end{array}\right)
$$

where variables $W_{j}$ are the amplitudes of relative displacements in the chain, variables $U_{j}$ are the amplitudes of displacement of the nonlinear oscillators, and $V$ is the amplitude of the main structure. 

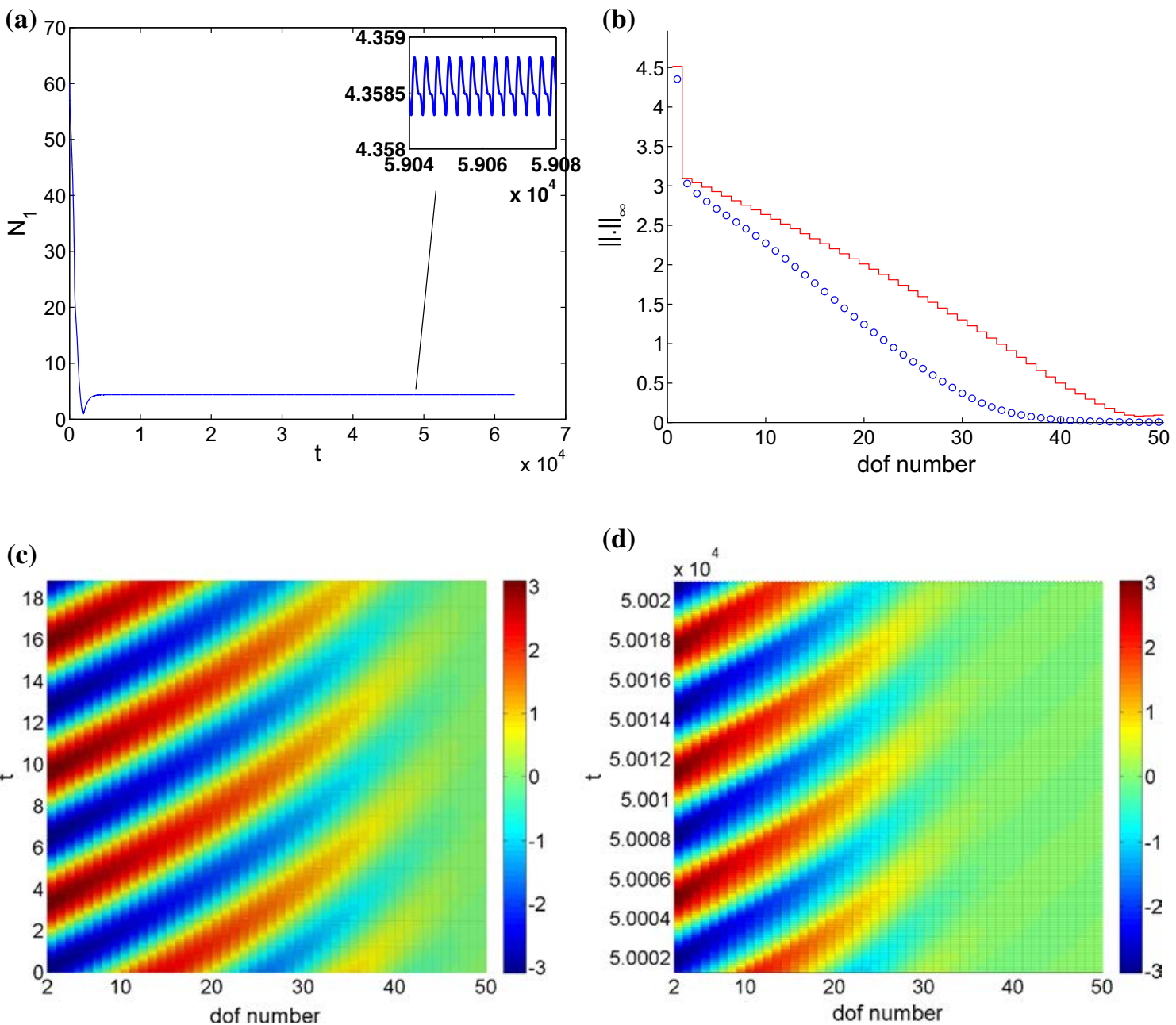

Fig. $4 N=50, \epsilon=0.001, \omega_{0}=1, r=5, c=0.2, B=2, c_{1}=4, D=50, \sigma=0$, and $f^{0}=15$-a $N_{1}$ versus time obtained from direct time integration of system (2.1), b comparison between system amplitudes obtained from analytical predictions corresponding to equilibrium point no. 1 (red lines) and numerical results at $\tau_{1}$ time scale with all harmonics (blue circles), c evolution of the amplitudes of oscillation of each mass of the chain obtained from analytical predictions (equilibrium point no. 1 ), $\mathbf{d}$ evolution of the amplitudes of oscillation of each mass of the chain obtained from numerical results at $\tau_{1}$ time scale (colour figure online)

Inserting (20) in (2.1) where damping and external excitation are set to zero and neglecting the third harmonic generated by cubic nonlinearities, one obtains the following system of algebraic equations:

$$
\left\{\begin{array}{c}
\left(\omega_{0}^{2}-\Omega^{2}\right) V-\epsilon r W_{1}=0 \\
\left((1+\epsilon) r-\Omega^{2}\right) W_{1}-\omega_{0}^{2} V-B W_{2}-\frac{3}{4} D W_{2}^{3}=0 \\
\left(2 B-\Omega^{2}\right) W_{2}-r W_{1}-B W_{3}+\frac{3}{4} D\left(2 W_{2}^{3}-W_{3}^{3}\right)=0 \\
\vdots \\
\left(2 B-\Omega^{2}\right) W_{j}-B\left(W_{j-1}+W_{j+1}\right)+\frac{3}{4} D\left(-W_{j-1}^{3}+2 W_{j}^{3}-W_{j+1}^{3}\right)=0 \\
\vdots \\
\left(2 B-\Omega^{2}\right) W_{N-1}-B W_{N-2}+\frac{3}{4} D\left(2 W_{N-1}^{3}-W_{N-2}^{3}\right)=0 .
\end{array}\right.
$$

This system of $N$ equations in $N+1$ unknowns enables to plot a representation of the nonlinear modes in the $\left(\Omega-W_{N-1}\right)$ plane, as shown in Fig. 9 where the parameters are the ones used in the previous Subsection for 


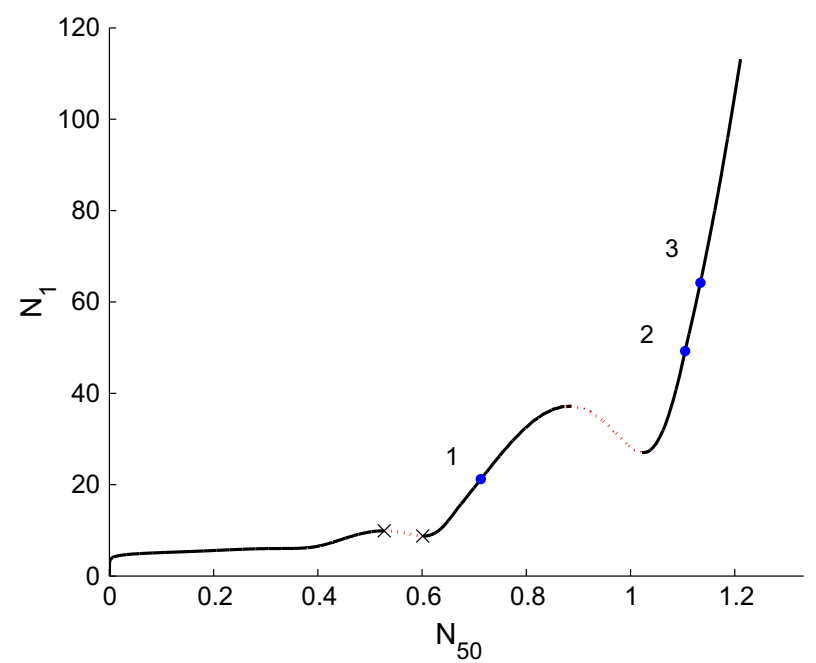

Fig. $5 N=50, \epsilon=0.001, \omega_{0}=1, r=5, c=0.2, B=2, c_{1}=4, D=50, \sigma=0$, and $f^{0}=60$-SIM of the system (black line) and position of equilibrium and singular points (blue points and black crosses, respectively). Red dotted lines denote unstable branches of the SIM. The system possesses three equilibrium points (no. 1, 2 and 3) and two singular points (colour figure online)

the three first examples. Figure 9b shows a zoomed-in view of Fig. 9a around $\Omega=1$, which is the excitation frequency considered in Sect. 4.1.

There are eleven solutions at $\Omega=1$, just above the horizontal line, corresponding to eleven possible excited modes at this frequency. Behaviors highlighted in examples 2 and 3 of Sect. 4.1 occur at $N_{50}=0.713$ and $N_{50}=1.188$, respectively. As a consequence, modal shapes at points $\mathrm{A}$ and B (see Fig. $9 \mathrm{~b}$ ) are expected to describe the general profiles depicted in Figs. 6 and 8. These modal shapes are represented in Fig. 10. They show a good qualitative agreement with analytical predictions (i.e., behaviors at equilibrium points), and numerical results exhibited previously. Therefore, we can assume that the system response at different levels of energy, i.e., for equilibrium points located on different branches of the SIM, is characterized by some of its nonlinear normal modes around the resonance of the main structure.

\subsection{Strongly modulated responses}

To highlight SMR, the following forcing parameters are used: $f^{0}=250$ and $\sigma=6$. The position of equilibrium and singular points around the SIM is depicted in Fig. 11a. The system possesses one unstable equilibrium point and six singular points. As a consequence, it will face SMR around one of the unstable zones of the SIM. A schematic representation of the latter behavior around unstable zone number (3) is described in Fig. $11 \mathrm{~b}$ (number (2) zone's size is not significant for the dynamics of the system). From initial conditions, the system moves toward the SIM and makes repeated bifurcations forming a closed-loop cycle. Nonetheless, more complicated cycles involving unstable zones number (1) and (3) could occur with the appropriate forcing amplitude.

Figure 12a, b plots $N_{1}$ versus time and the evolution of the behavior of the chain obtained from numerical simulations. It appears that the system is facing SMR around the third unstable zone. Time series of several oscillators of the chain depicted in Fig. 13, where the final time corresponds to the one of Fig. 12b, show that this behavior lasts for a long time. During a bifurcation, the chain of nonlinear oscillators suddenly switches from one modal behavior to another (see Fig. 12b). These behaviors actually correspond to the ones which the system would face if it had reached an equilibrium point on the corresponding branch of the SIM, namely behaviors described in the last two examples of Sect. 4.1 (see Figs. 6, 8). Thus, we can define SMR here as persistent bifurcations between nonlinear normal modes of the chain of oscillators. In this case, two modes are involved. However, in the presence of more complex geometries of the SIM, and convoluted SMR cycles as described above, three or more modes could appear in these quasi-periodic regimes. 

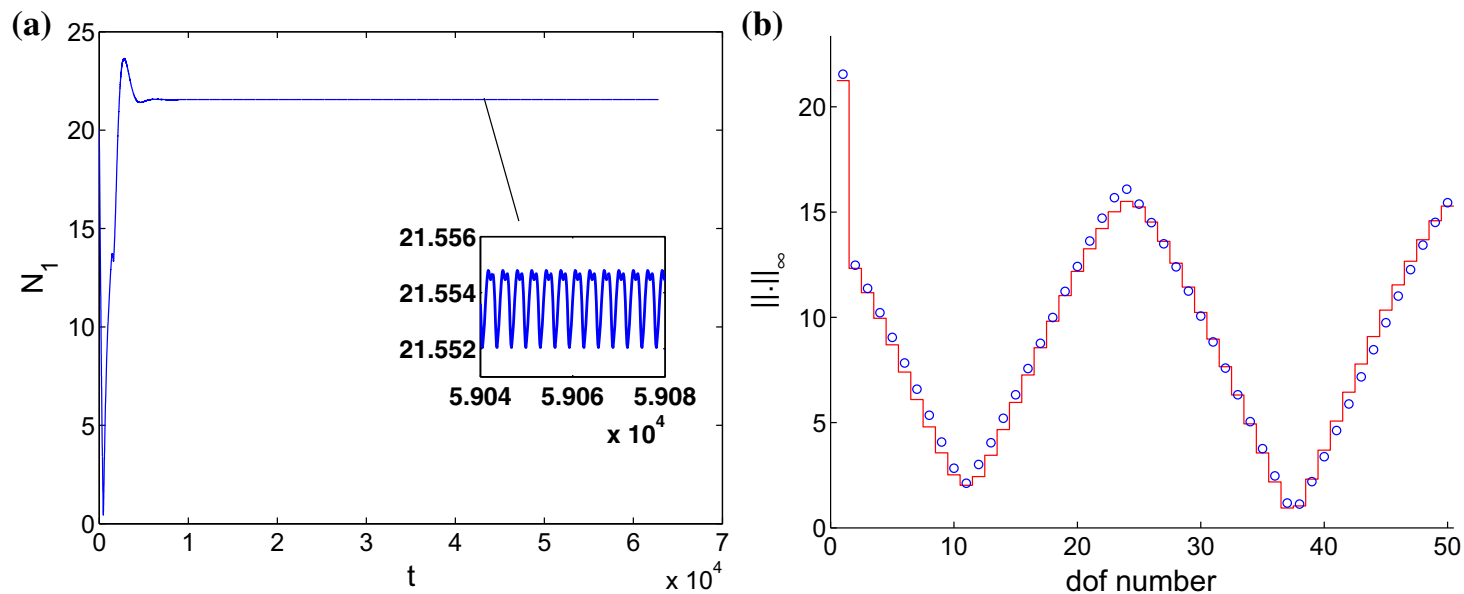

(c)

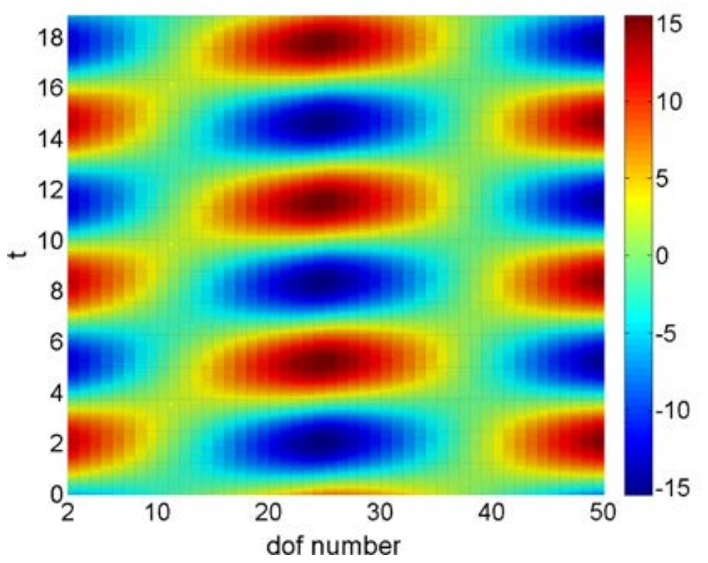

(d)

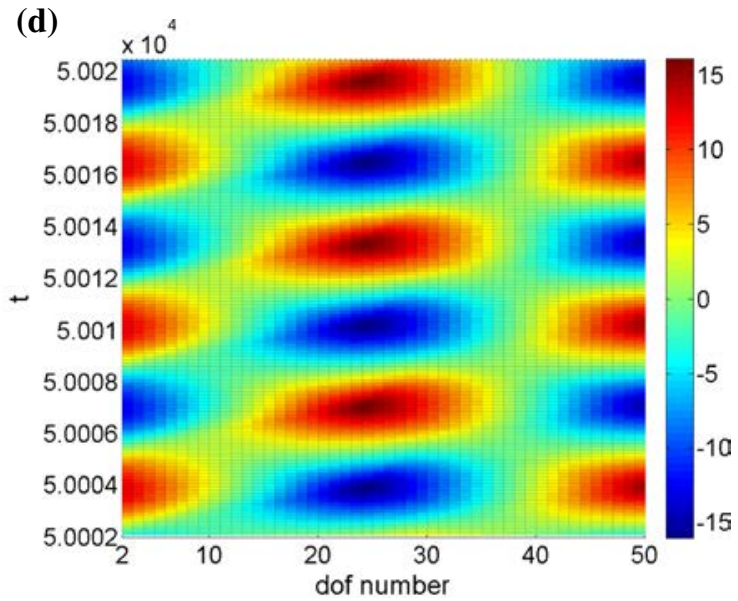

Fig. $6 N=50, \epsilon=0.001, \omega_{0}=1, r=5, c=0.2, B=2, c_{1}=4, D=50, \sigma=0$, and $f^{0}=60$ - a $N_{1}$ versus time obtained from direct time integration of system (2.1), b comparison between system amplitudes obtained from analytical predictions corresponding to equilibrium point no. 1 (red lines) and numerical results at $\tau_{1}$ time scale with all harmonics (blue circles), $\mathbf{c}$ evolution of the amplitudes of oscillation of each mass of the chain obtained from analytical predictions (equilibrium point no. 1 ), $\mathbf{d}$ evolution of the amplitudes of oscillation of each mass of the chain obtained from numerical results at $\tau_{1}$ time scale (colour figure online)

\section{Application to control}

Investigation of equilibrium and singular points and design of geometry of the SIM are linked to problems of passive control of structural systems by addition of a nonlinear chain. The geometry of the SIM provides all possible energy paths which the overall system can experience during interaction between its oscillators while equilibrium and singular points give information about all possible periodic regimes or SMR at slow time scale(s).

This Section aims to give evidence of passive control of the main system considering the same parameters as in Sect. 4. To this end, six amplitude-frequency graphs (i.e., $N_{1}-\sigma$ graphs) corresponding to six different forcing amplitudes are plotted in Fig. 14. Stable and unstable equilibrium points are illustrated in blue and red points, while singular points are marked by black dashed-dotted lines. Furthermore, the maximum amplitude that the main system would face without the chain of oscillators, called as "maximum linear amplitude", is written in each Figure. This is actually the amplitude of the linear system at the exact resonance, i.e., at $\sigma=0$. At low forcing amplitude $f^{0}=15$ (see Fig. 14a), it is clear that a major part of vibratory energy has been transferred in the chain as the maximum amplitude of the main system in the presence of the chain is equal to $6.13 \%$ of the maximum linear amplitude. Increasing the value of $f^{0}$ to 28 (see Fig. 14b), an isolated branch appears and reduces the control efficiency as the maximum amplitude raises to $13.95 \%$ of the maximum linear 


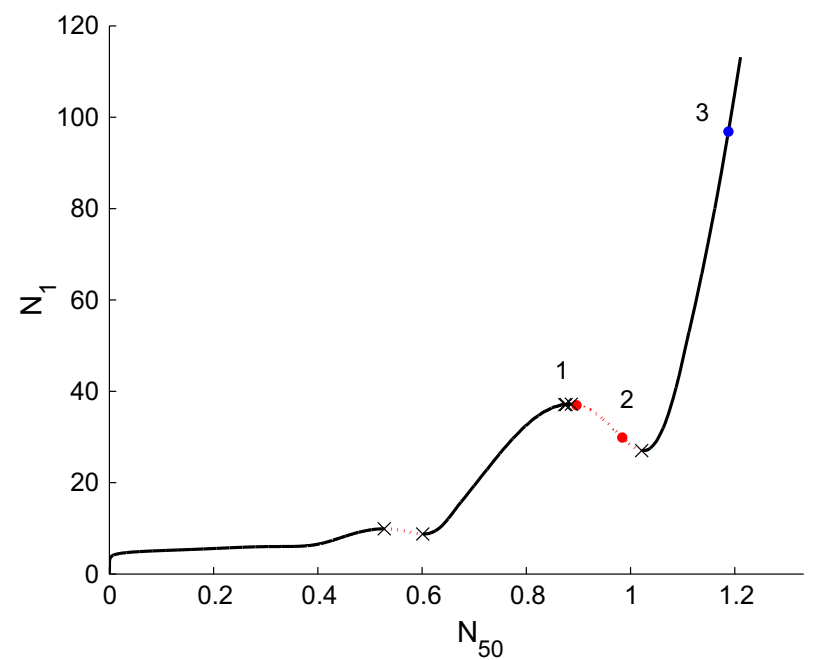

Fig. $7 N=50, \epsilon=0.001, \omega_{0}=1, r=5, c=0.2, B=2, c_{1}=4, D=50, \sigma=0$, and $f^{0}=280$-SIM of the system (black line) and position of stable/unstable equilibrium points (blue/red points) and singular points (black crosses). Red dotted lines denote unstable branches of the SIM. The system possesses one stable (no. 1) and two unstable equilibrium points (no. 2 and 3 ) and six singular points (colour figure online)

amplitude. This value reaches $26.36 \%$ at $f^{0}=60$ as singular points appear and then drops to $20.16,14.65$, and $11.02 \%$ for the cases $f^{0}=90, f^{0}=150$, and $f^{0}=250$, respectively (see Fig. 14c-f). Besides, for the four latter cases, the presence of singular points can lead to SMR, depending on initial conditions and distribution of equilibrium points, and thus confine the oscillations of the main system between $N_{1}$-amplitudes of the black dashed-dotted lines.

As a summary, considering given detuning parameter $\sigma$ and forcing the amplitude $f^{0}$ intervals, i.e., from -10 to $10\left(-15\right.$ to 15 for $\left.f^{0}=250\right)$ and from 15 to 250 , respectively, the amplitude of the linear system has been significantly reduced by addition of a chain of nonlinear oscillators and divided by almost 4 in the worst case.

\section{Multi-degree-of-freedom main structure}

\subsection{Analytical developments}

All developments of the paper have been based on the consideration of an SDOF main system. However, this SDOF system can be supposed as the result of the projection of the dynamics of an MDOF main structure on one of its modes of interest (to be controlled or harvested). Let us consider the system presented in Fig. 15, which consists of $n$ forced linear oscillators in series coupled to the nonlinear chain presented in Sect. 2, with the restoring force $V(z)=\epsilon\left(B z+D z^{3}\right)$ and mass $\epsilon$ for each oscillator.

The governing equations of the $n$ linear structures read:

$$
\mathbf{M} \ddot{\mathbf{X}}+\epsilon \mathbf{C} \dot{\mathbf{X}}+\mathbf{K X}+\epsilon \mathbf{K}_{1}\left(\mathbf{X}-\mathbf{U}_{1}\right)+\epsilon \mathbf{A}_{1}\left(\dot{\mathbf{X}}-\dot{\mathbf{U}}_{1}\right)=\epsilon \hat{\mathbf{F}} \sin (\tilde{\omega} t)
$$

where

$$
\mathbf{M}=\left(\begin{array}{cccc}
M_{1} & 0 & \ldots & 0 \\
0 & \ddots & \ddots & \vdots \\
\vdots & \ddots & \ddots & 0 \\
0 & \ldots & 0 & M_{n}
\end{array}\right), \mathbf{K}=\left(\begin{array}{ccccc}
K_{1}+K_{2} & -K_{2} & 0 & \ldots & 0 \\
-K_{2} & \ddots & \ddots & & \vdots \\
0 & \ddots & \ddots & \ddots & 0 \\
\vdots & \ddots & \ddots & K_{n-1}+K_{n} & -K_{n} \\
0 & \ldots & 0 & -K_{n} & K_{n}
\end{array}\right)
$$


(a)

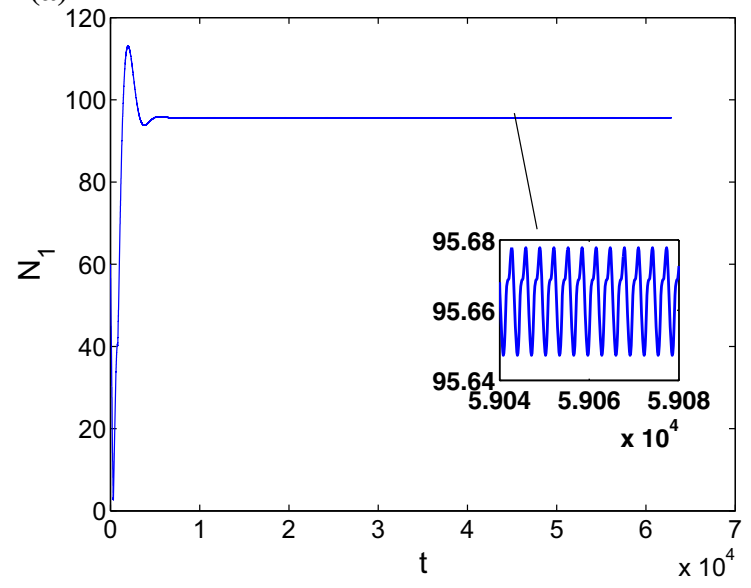

(c)

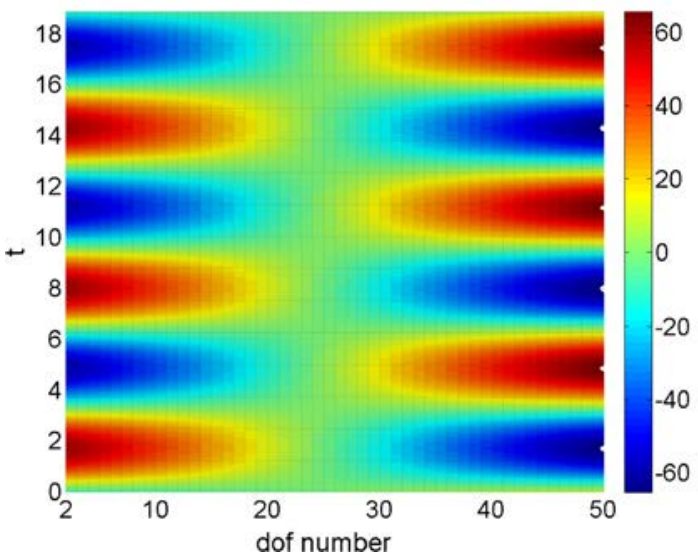

(b)

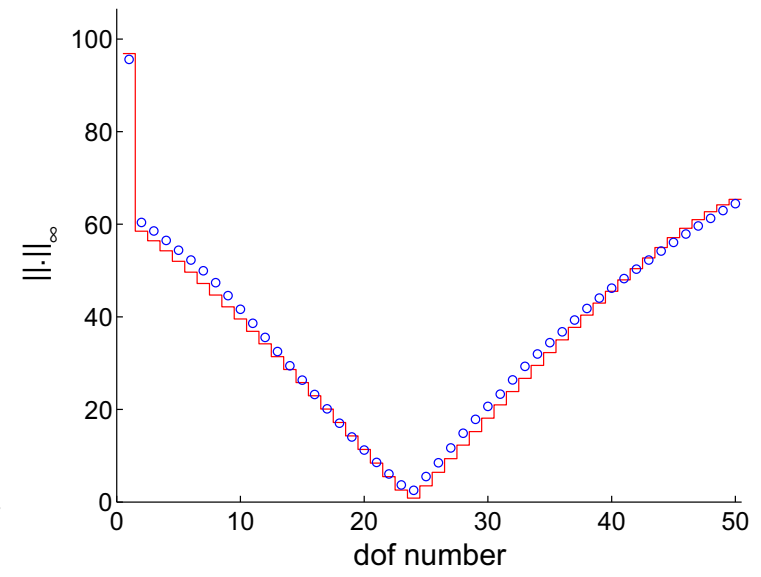

(d)

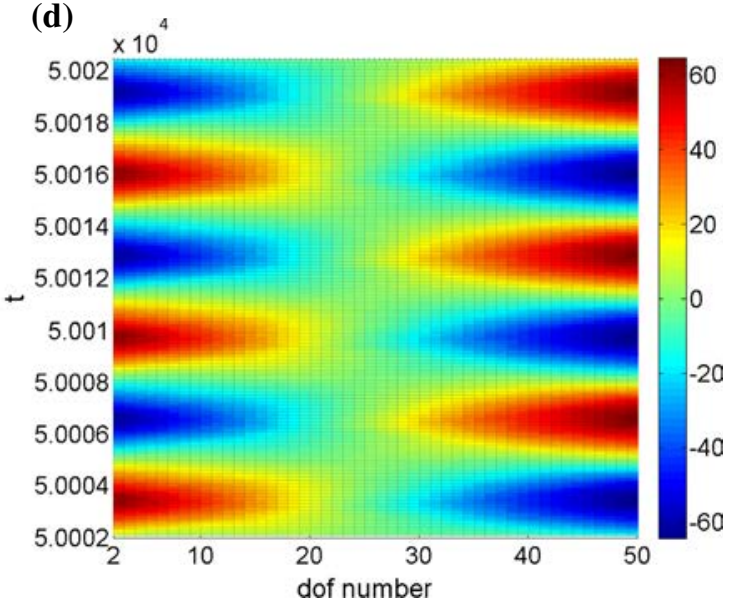

Fig. $8 N=50, \epsilon=0.001, \omega_{0}=1, r=5, c=0.2, B=2, c_{1}=4, D=50, \sigma=0$, and $f^{0}=280$-a $N_{1}$ versus time obtained from direct time integration of system (2.1), b comparison between system amplitudes obtained from analytical predictions corresponding to equilibrium point no. 3 (red lines) and numerical results at $\tau_{1}$ time scale with all harmonics (blue circles), c evolution of the amplitudes of oscillation of each mass of the chain obtained from analytical predictions (equilibrium point no. 3 ), $\mathbf{d}$ evolution of the amplitudes of oscillation of each mass of the chain obtained from numerical results at $\tau_{1}$ time scale (colour figure online)

(a)

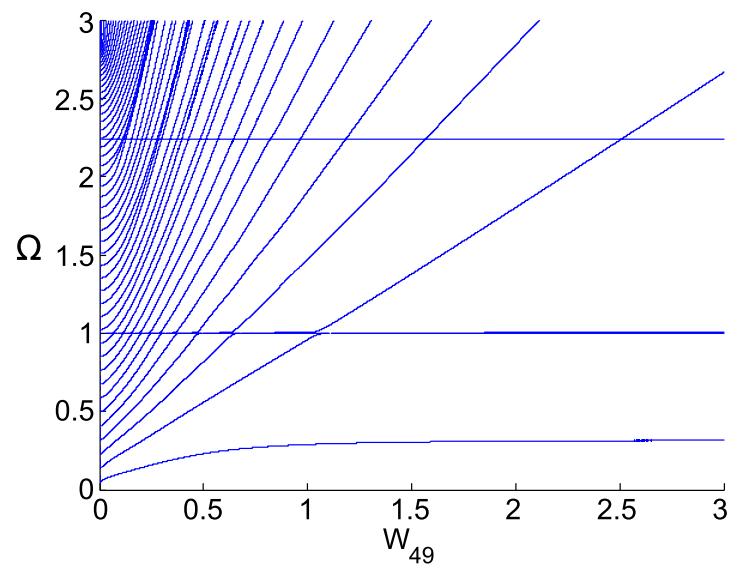

(b)

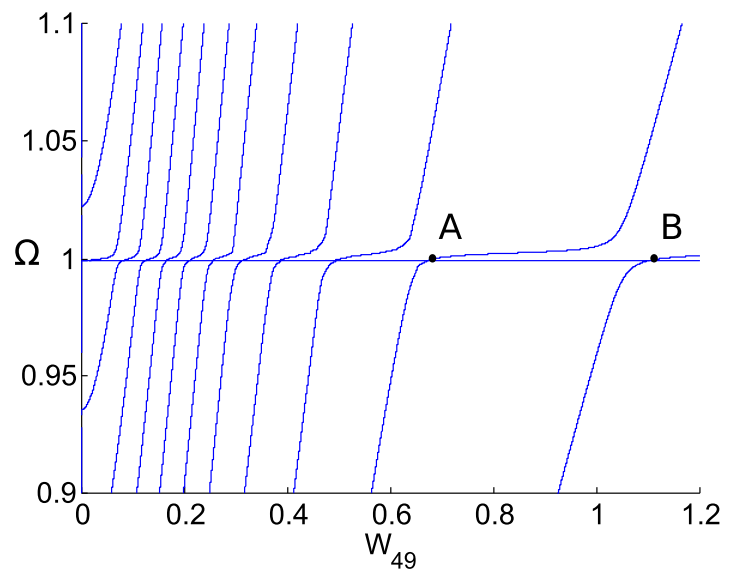

Fig. $9 N=50, \epsilon=0.001, \omega_{0}=1, r=5, B=2$, and $D=50$-a representation of the nonlinear normal modes of the system in the $\left(\Omega-W_{N-1}\right)$ plane, $\mathbf{b}$ zoomed-in view around $\Omega=1$ 
(a)

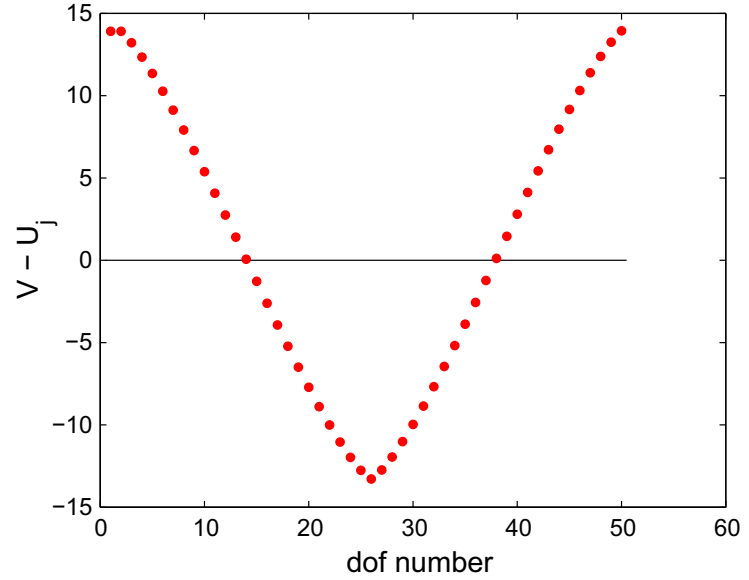

(b)

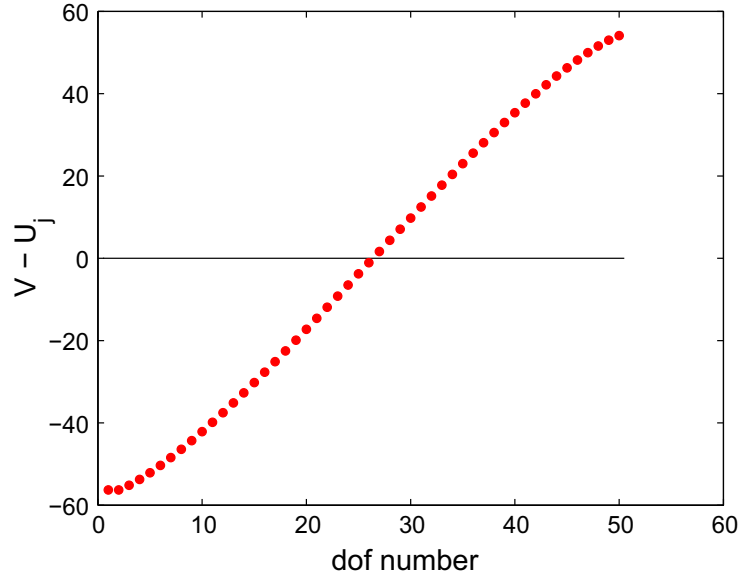

Fig. $10 N=50, \epsilon=0.001, \omega_{0}=1, r=5, B=2, D=50$, and $\Omega=1$-modal shape of the system at points A and B (see Fig. 9b). a Point A, b Point B

(a)

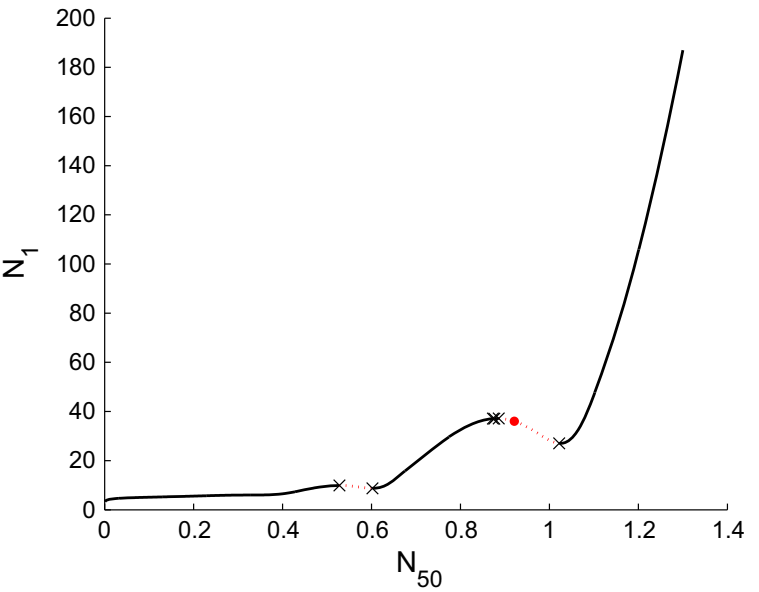

(b)

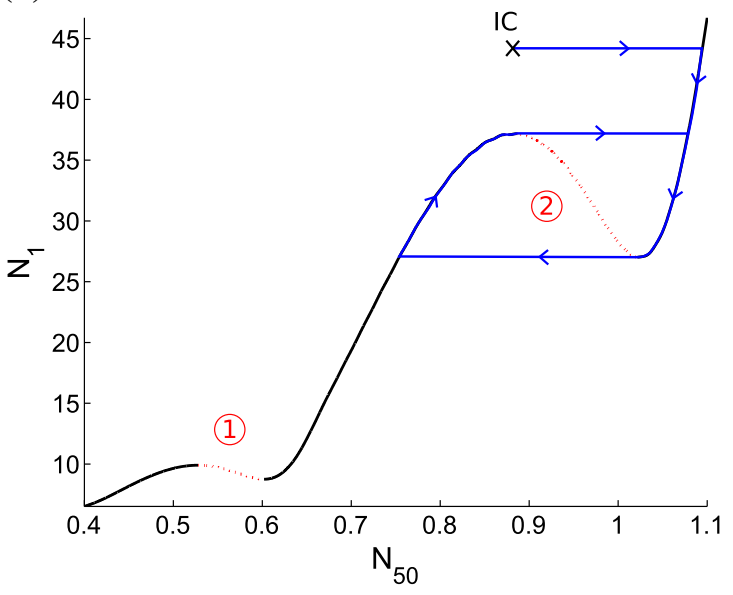

Fig. $11 N=50, \epsilon=0.001, \omega_{0}=1, r=5, c=0.2, B=2, c_{1}=4, D=50, \sigma=6$, and $f^{0}=250$ - a SIM of the system (black line) and position of the unstable equilibrium point (red point) and singular points (black crosses). Red dotted lines denote unstable branches of the SIM. The system possesses one unstable equilibrium point and six singular points, $\mathbf{b}$ schematic representation of the system behavior during SMR. "IC" "initial conditions," while the circled red numbers enumerate the unstable zones (colour figure online)

$$
\begin{aligned}
& \mathbf{C}=\left(\begin{array}{ccccc}
C_{1}+C_{2} & -C_{2} & 0 & \ldots & 0 \\
-C_{2} & \ddots & \ddots & & \vdots \\
0 & \ddots & \ddots & \ddots & 0 \\
\vdots & \ddots & \ddots & C_{n-1}+C_{n}-C_{n} \\
0 & \ldots & 0 & -C_{n} & C_{n}
\end{array}\right), \mathbf{K}_{1}=\left(\begin{array}{cccc}
0 \ldots & \ldots & 0 \\
\vdots & \ddots & & \vdots \\
0 & \ldots & 0 & 0 \\
0 & \ldots & 0 & k
\end{array}\right), \\
& \mathbf{A}_{1}=\left(\begin{array}{cccc}
0 & \ldots & \ldots & 0 \\
\vdots & \ddots & & \vdots \\
0 & \ldots & 0 & 0 \\
0 & \ldots & 0 & a
\end{array}\right), \mathbf{X}=\left(\begin{array}{c}
X_{1} \\
\vdots \\
X_{n}
\end{array}\right), \mathbf{U}_{1}=\left(\begin{array}{c}
0 \\
\vdots \\
0 \\
u_{1}
\end{array}\right), \hat{\mathbf{F}}=\left(\begin{array}{c}
F_{1} \\
\vdots \\
F_{n}
\end{array}\right)
\end{aligned}
$$

In order to compute the normal modes of the $n$-DOF linear system, we search for eigenvalues and eigenvectors of the matrix $\mathbf{M}^{-1} \mathbf{K}$, namely $\left(\omega_{1}^{2}, \ldots, \omega_{n}^{2}\right)$ and $\mathbf{P}=\left(\mathbf{P}_{1}, \ldots, \mathbf{P}_{n}\right)$ where $\mathbf{P}$ is the matrix of the modal 

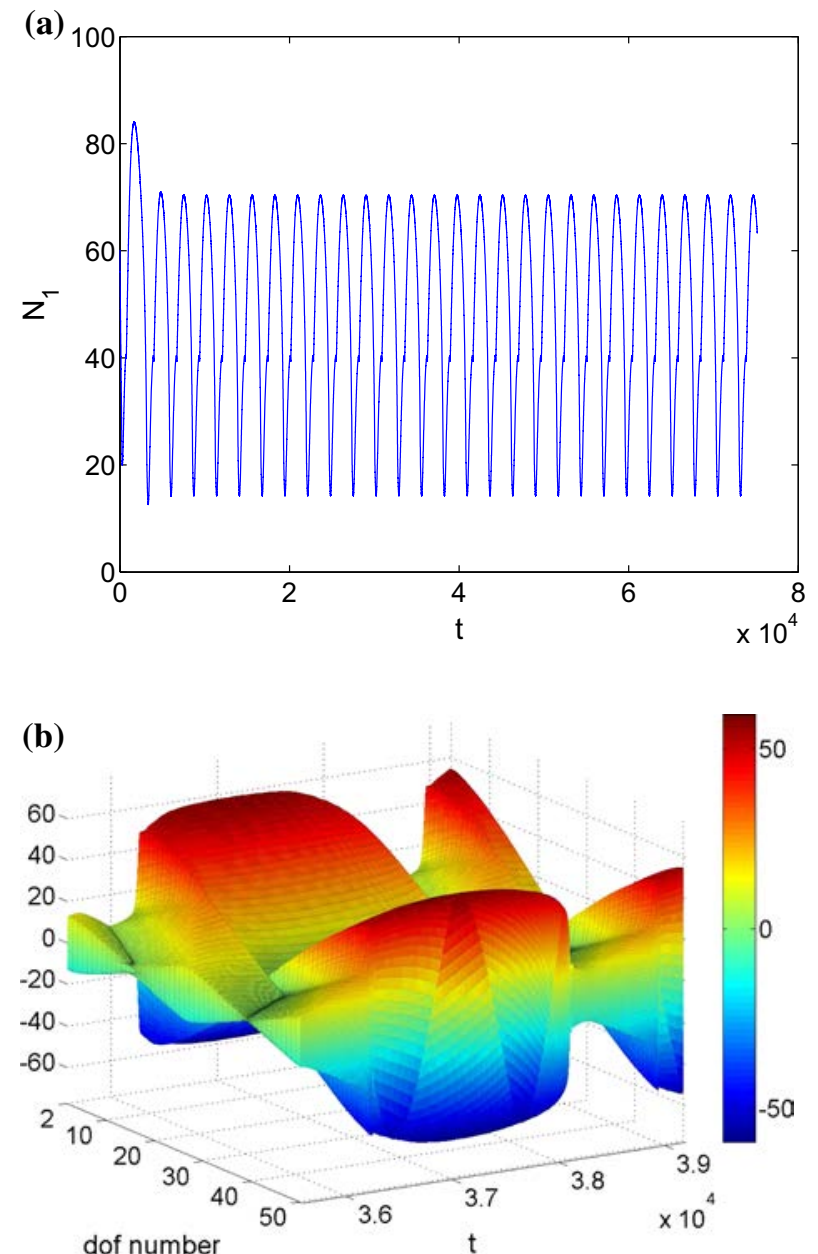

Fig. $12 N=50, \epsilon=0.001, \omega_{0}=1, r=5, c=0.2, B=2, c_{1}=4, D=50, \sigma=6$, and $f^{0}=250-\mathbf{a} N_{1}$ versus time obtained from direct numerical integration of system (2.1), $\mathbf{b}$ evolution of the amplitudes of oscillation of each mass of the chain obtained from numerical results during SMR

basis. Defining the modal coordinates $\mathbf{Q}=\left(Q_{1}, \ldots, Q_{n}\right)$ as $\mathbf{X}=\mathbf{P Q}$, one can obtain the following system:

$$
\begin{aligned}
& \ddot{\mathbf{Q}}+\epsilon \tilde{\mathbf{C}} \dot{\mathbf{Q}}+\tilde{\mathbf{K}} \mathbf{Q}+\epsilon \mathbf{P}^{-1} \mathbf{M}^{-1} \mathbf{K}_{1}\left(\mathbf{P Q}-\mathbf{U}_{1}\right) \\
& \quad+\epsilon \mathbf{P}^{-1} \mathbf{M}^{-1} \mathbf{A}_{1}\left(\mathbf{P} \mathbf{Q}-\dot{\mathbf{U}}_{1}\right)=\epsilon \mathbf{P}^{-1} \mathbf{M}^{-1} \hat{\mathbf{F}} \sin (\tilde{\omega} t) \\
& \text { where } \\
& \tilde{\mathbf{C}}=\mathbf{P}^{-1} \mathbf{M}^{-1} \mathbf{C P} \tilde{\mathbf{K}}=\mathbf{P}^{-1} \mathbf{M}^{-1} \mathbf{K} \mathbf{P} .
\end{aligned}
$$

$\tilde{\mathbf{K}}$ is a diagonal matrix whose elements are $\left(\omega_{1}^{2}, \ldots, \omega_{n}^{2}\right)$. Furthermore, we assume that $\tilde{\mathbf{C}}$ is also diagonal (Basile hypothesis), with diagonal elements $\left(\tilde{C}_{1}, \ldots, \tilde{C}_{n}\right)$.

Now that the system has been projected in the modal basis, we assume that we excite the system around the frequency of the $l$ th mode, i.e., $\tilde{\omega}^{2}=\omega_{l}^{2}(1+\tilde{\sigma} \epsilon)$. Then, two questions need to be addressed:

- Given the frequency of solicitation, one expects to excite only the $l$ th mode of the system. Will the other modes be excited too because of the coupling with the chain?

- Can the behavior of the overall system be predicted by the model developed in Sects. 2 and 3? 

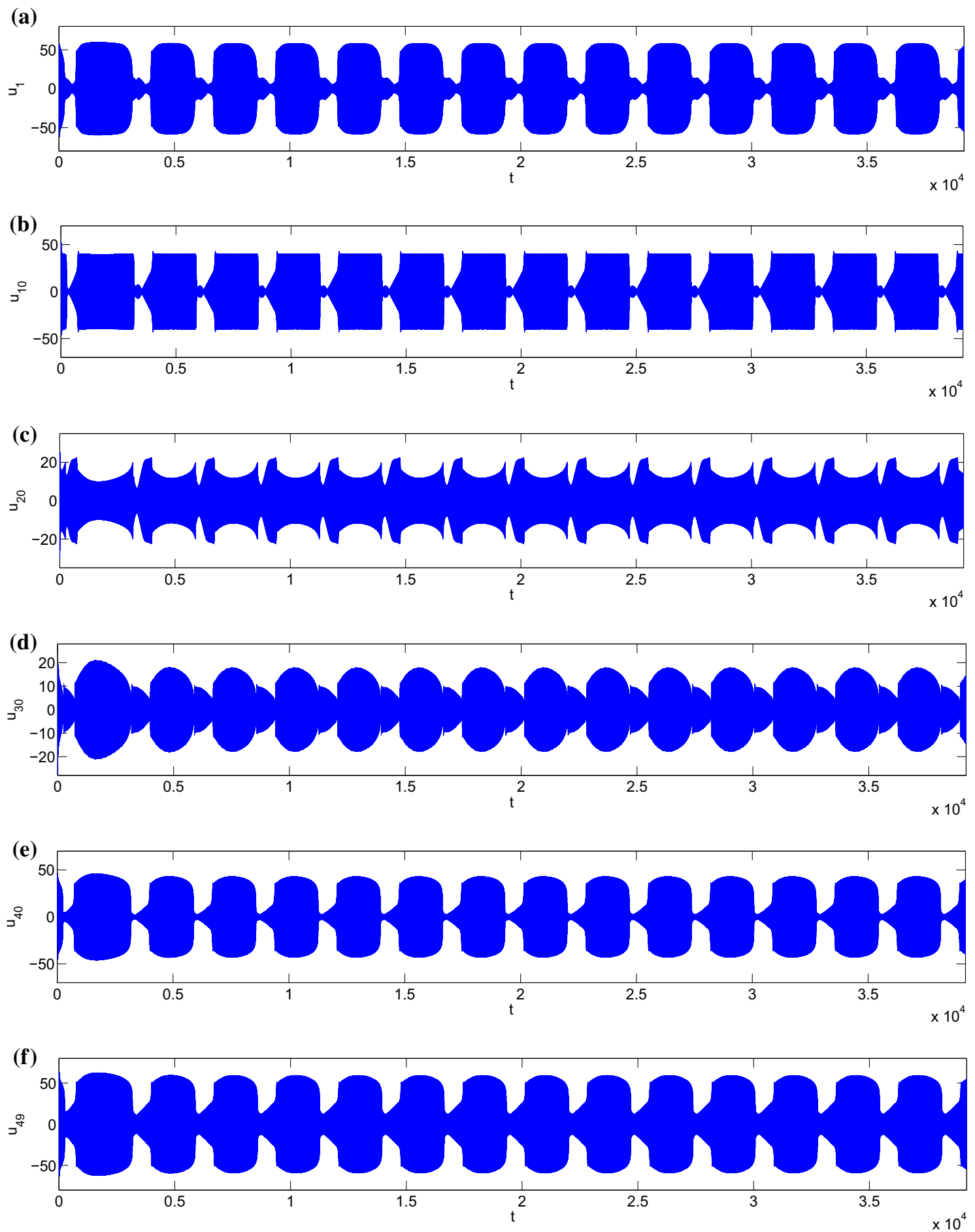

Fig. $13 N=50, \epsilon=0.001, \omega_{0}=1, r=5, c=0.2, B=2, c_{1}=4, D=50, \sigma=6$, and $f^{0}=250$-time series of several oscillators of the chain obtained from direct numerical integration of system (2.1) during SMR: a $u_{1}, \mathbf{b} u_{10}, \mathbf{c} u_{20}, \mathbf{d} u_{30}, \mathbf{e} u_{40}$, f $u_{49}$ 

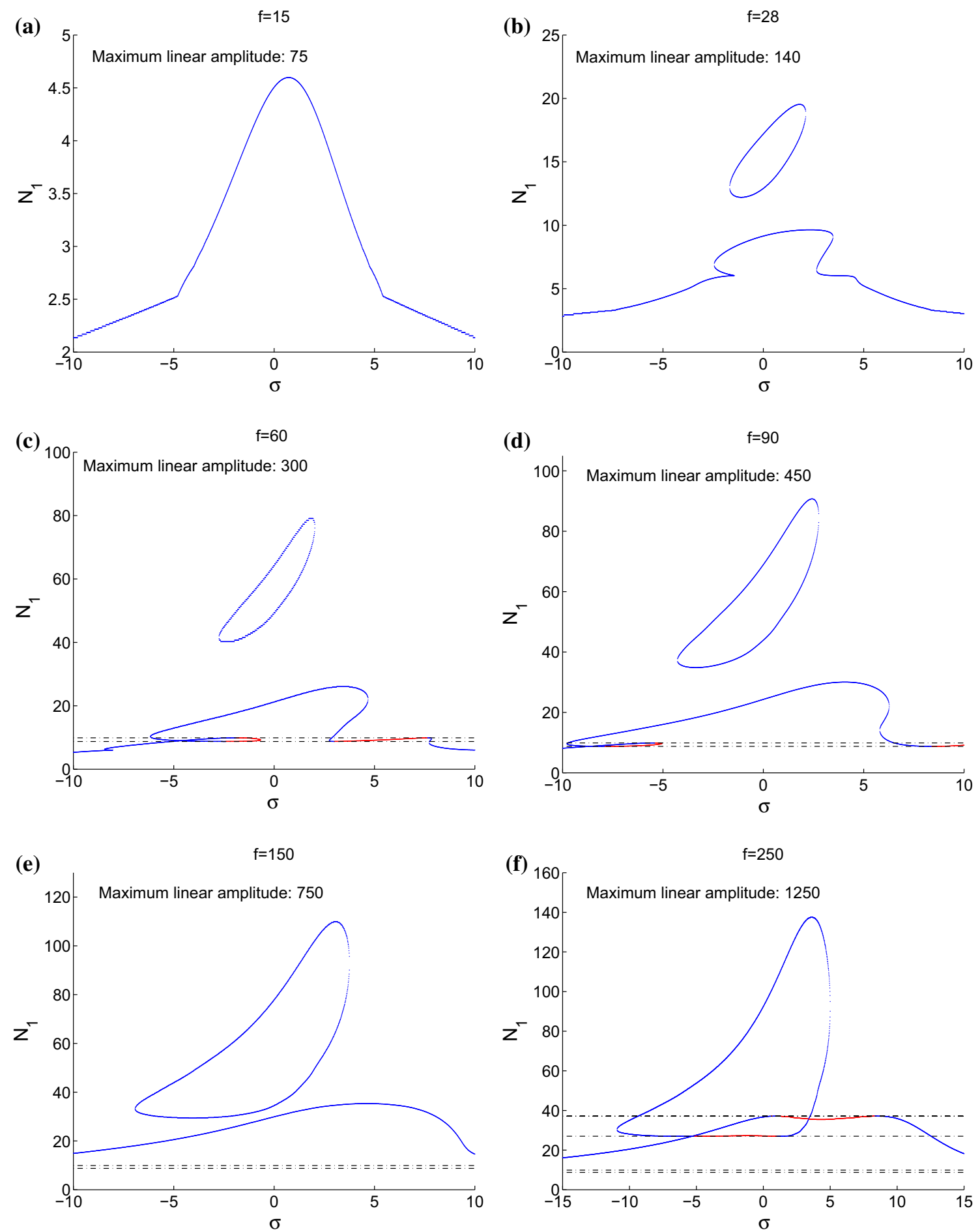

Fig. 14 Amplitude-frequency graphs considering the following parameters: $N=50, \epsilon=0.001, \omega_{0}=1, r=5, c=0.2, B=2$, $c_{1}=4, D=50-\mathbf{a} f^{0}=15, \mathbf{b} f^{0}=28$, c $f^{0}=60$, d $f^{0}=90$, e $f^{0}=150$, $\mathbf{f} f^{0}=250$. Blue and red points depict stable and unstable equilibrium points, while black dashed-dotted lines denote the presence of singular points (colour figure online) 


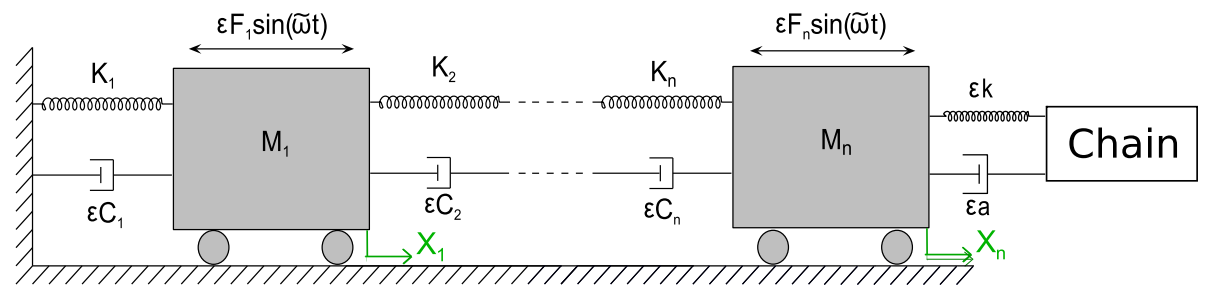

Fig. 15 MDOF forced main structure coupled to a chain of nonlinear oscillators (see Fig. 1 for details of the chain)

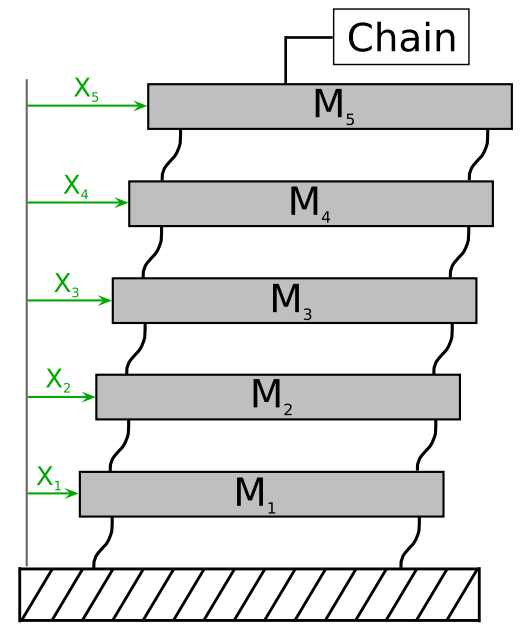

Fig. 16 Model of a five-story building with a chain of nonlinear oscillators attached on its roof

Table 2 Values of the parameters chosen for the numerical simulation performed on a five-DOF linear main structure coupled to a chain of nonlinear oscillators

\begin{tabular}{lllll}
\hline$M_{1}$ & $M_{2}$ & $M_{3}$ & $M_{4}$ & $M_{5}$ \\
0.5 & 1.5 & 1 & 0.5 & 1 \\
$K_{1}$ & $K_{2}$ & $K_{3}$ & $K_{4}$ & $K_{5}$ \\
2 & 1 & 1.5 & 1.413 & 2 \\
$C_{1}$ & $C_{2}$ & $C_{3}$ & $C_{4}$ & $C_{5}$ \\
0.4 & 0.2 & 0.3 & 0.28 & 0.4 \\
\hline
\end{tabular}

Introducing $\tilde{M}_{i j}$ and $P_{i j}$ as the elements of the $i$ th line and the $j$ th column of matrices $\mathbf{P}^{-1} \mathbf{M}^{-1}$ and $\mathbf{P}$, respectively, Eq. (24) reads as the following system of $n$ linear equations:

$$
\begin{aligned}
& \ddot{Q}_{j}+\epsilon \tilde{C}_{j} \dot{Q}_{j}+\omega_{j}^{2} Q_{j}+\epsilon \tilde{M}_{j n}\left[\sum_{i=1}^{n} P_{n i}\left(k Q_{i}+a \dot{Q}_{i}\right)-\left(k u_{1}+a \dot{u}_{1}\right)\right] \\
& =\epsilon \sum_{i=1}^{n} \tilde{M}_{j i} F_{i} \sin \left(\omega_{l} t \sqrt{1+\tilde{\sigma} \epsilon}\right) \quad j=1, \ldots, n
\end{aligned}
$$

We assume that the modes are well separated, i.e., the angular frequencies $\omega_{j}$ are not too close from each other. As $u_{1}$ and $\dot{u}_{1}$ are supposed to vibrate at the forcing frequency (see Sect. 2), it seems reasonable to assume that the dynamics of the $n$-DOF linear system will be mainly described by its $l$ th mode. This assumption will be verified thanks to numerical simulations in Sect. 6.2. As a consequence, we consider that all other modes will have a small contribution in the response of the overall system, i.e., 
(a)

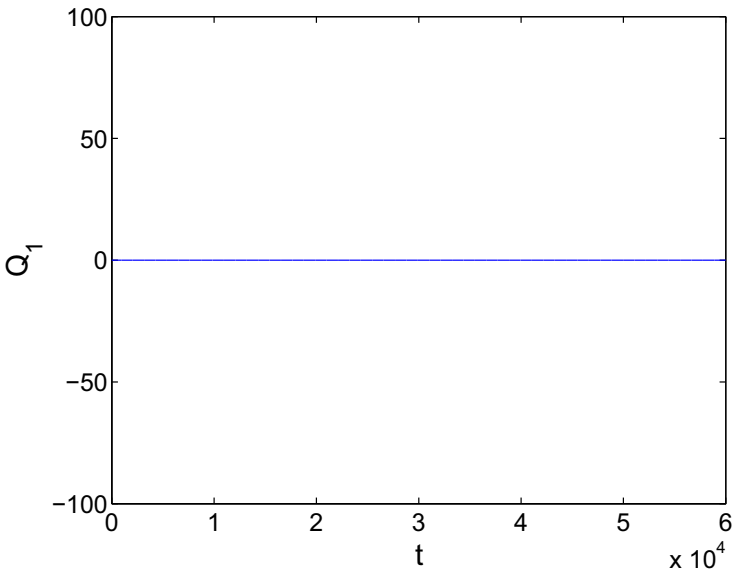

(c)

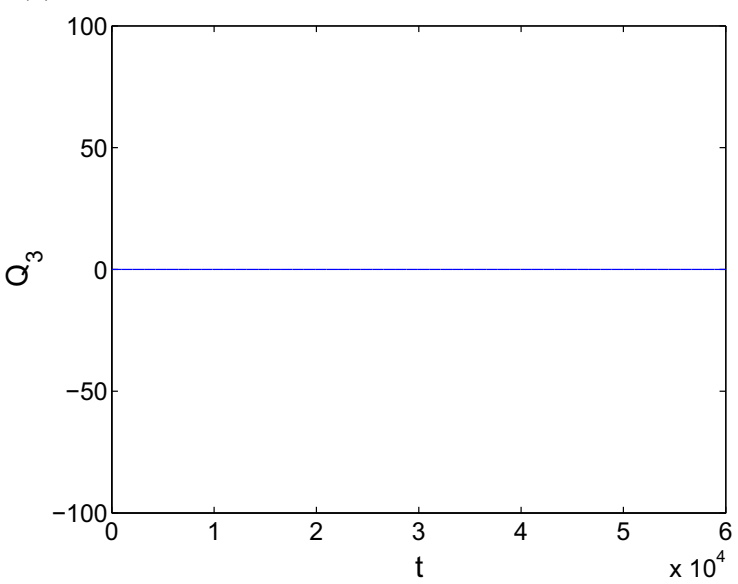

(e)

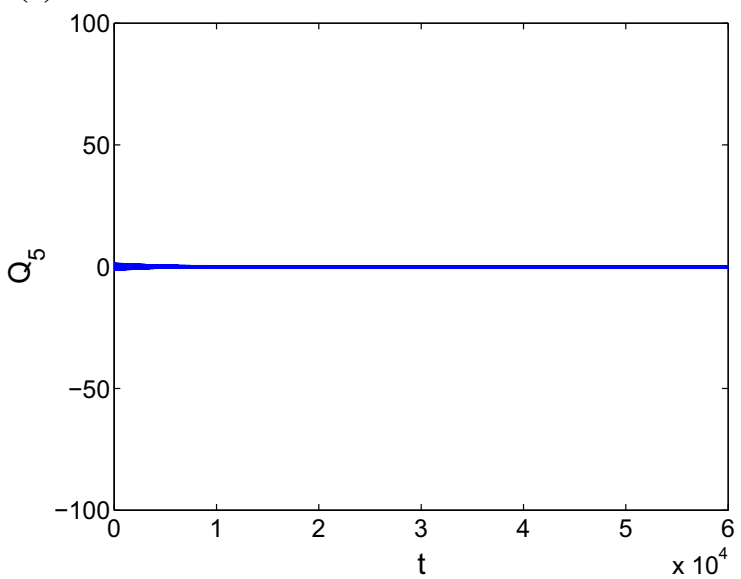

(b)

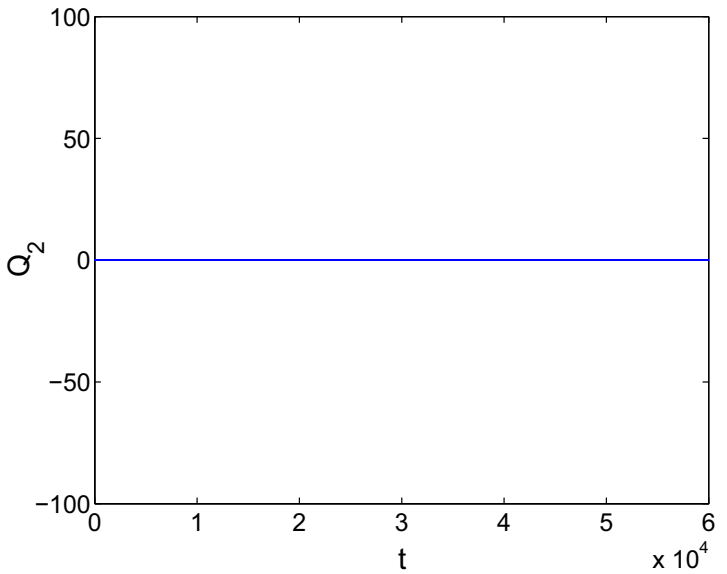

(d)

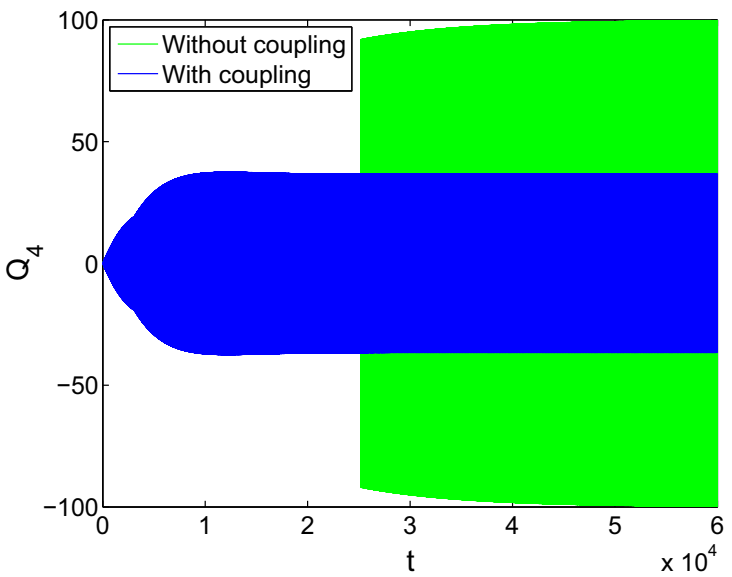

(f)

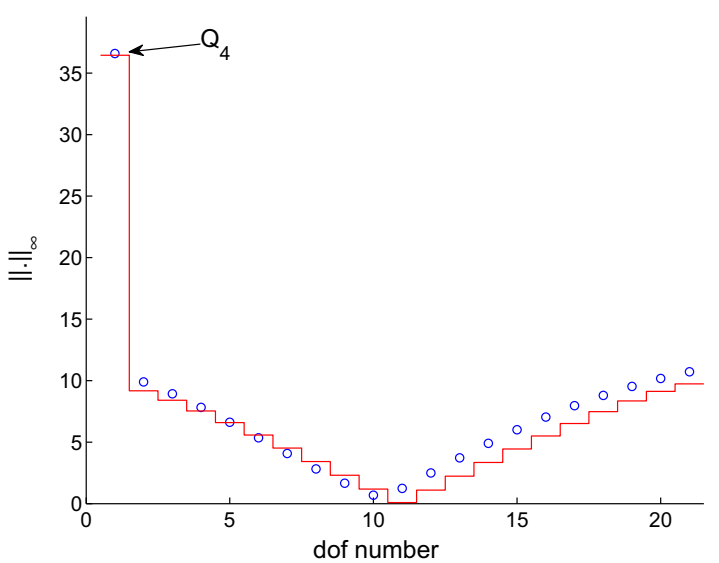

Fig. $17 \epsilon=0.001, k=0.8, a=1.3, B=2, D=50, \tilde{\sigma}=0$, and $F_{i}=-150.7, \forall i-\mathbf{a}-\mathbf{e}$ evolution of modal coordinates $Q_{j}$ versus time obtained from direct numerical integration of system (22) (blue lines). In d, evolution of $Q_{4}$ without coupling of the chain is depicted in green - $\mathbf{f}$ comparison of system amplitudes obtained from analytical predictions (red lines) and numerical results at $\tau_{1}$ time scale with all harmonics (blue circles) (colour figure online) 


$$
Q_{j}(t)=\mathcal{O}(\epsilon) \quad \forall j \in[|1, n|] \backslash\{l\} .
$$

Neglecting terms of $\epsilon^{2}$ order, the $l$ th equation of system (25) reads:

$$
\begin{aligned}
& \ddot{Q}_{l}+\epsilon \tilde{C}_{l} \dot{Q}_{l}+\omega_{l}^{2} Q_{l}+\epsilon \tilde{M}_{l n}\left[P_{n l}\left(k Q_{l}+a \dot{Q}_{l}\right)-\left(k u_{1}+a \dot{u}_{1}\right)\right] \\
& =\epsilon \sum_{i=1}^{n} \tilde{M}_{l i} F_{i} \sin \left(\omega_{l} t \sqrt{1+\tilde{\sigma} \epsilon}\right) .
\end{aligned}
$$

Equation (27) has the same form as Eq. (2.1). Thus, we can identify the expressions of the parameters of the latter equation as:

$$
\left\{\begin{array}{l}
v=P_{n l} Q_{l}, \\
\omega_{0}^{2}=\omega_{l}^{2}, \\
r=\tilde{M}_{l n} P_{n l} k \\
c=\tilde{C}_{l}, \\
c_{1}=\tilde{M}_{l n} P_{n l} a, \\
f^{0}=P_{n l} \sum_{i=1}^{n} \tilde{M}_{l i} F_{i}, \\
\sigma=\tilde{\sigma} .
\end{array}\right.
$$

We have obtained the rescaled parameters that should be used to implement the analytical method described in Sects. 2 and 3.

\subsection{Numerical verifications}

In order to confirm the assumptions made in Sect. 6.1, a system consisting of a five degree-of-freedom linear structure coupled to 20 nonlinear oscillators in series is investigated. Such system could be used to model, for instance, a five-story building with a chain of 20 nonlinear oscillators attached on its roof in order to control one of its modes. Figure 16 depicts an idealized model of such building that corresponds to the model described in Sect. 6.1 with $n=5$. Analytical predictions stemming from Sect. 2 and the 3 and the design procedure of Sect. 6.1 are compared with results obtained from direct numerical integration of system (22).

The values of the parameters relative to the main system are given in Table 2. One can compute the normal modes of the system and obtain the following angular frequencies: $\omega_{1}=2.95, \omega_{2}=2.51, \omega_{3}=1.80, \omega_{4}=1$, and $\omega_{5}=0.36$. The system is excited around the fourth mode, i.e., $\tilde{\omega}^{2}=\omega_{4}^{2}(1+\tilde{\sigma} \epsilon)$. All remaining parameters are chosen as follows: $\epsilon=0.001, k=0.8, a=1.3, B=2, D=50, \tilde{\sigma}=0$, and $F_{i}=-150.7, \forall i$. All masses are at rest at initial time. Rescaled parameters as defined in Eq. (28) are used for analytical predictions. Figure 17 a-e plots the evolution of modal coordinates $Q_{j}$ over time. Only the fourth mode is excited, while the other modes remain almost at rest, which validates the hypothesis made in Sect. 6.1. Moreover, the evolution of $Q_{4}$ versus time without addition of the chain is plotted in Fig. 17d. The maximum amplitude obtained is about three times larger than the amplitude obtained with coupling of the nonlinear chain. Since only one mode is excited, the amplitudes of displacement of each mass of the main system are proportional to $Q_{4}$, meaning that displacements of each DOF have been divided by 3. Finally, Fig. 17f compares the system amplitudes obtained from numerical simulation with results obtained from analytical developments which predict that the system will reach a periodic regime at $\tau_{1}$ time scale whose amplitudes are represented by red lines. Once again, analytical developments are in good agreement with numerical results.

\section{Conclusions}

A general methodology of the analytical treatment of an $N$-degree-of-freedom system, consisting of a linear structure linearly coupled to a chain of $(N-1)$ nonlinear oscillators, is presented. Time multi-scale behaviors of the system are investigated: the study at fast time scale provides the slow invariant manifold of the system, while equilibrium and singular points, corresponding to periodic regimes and strongly modulated responses, respectively, are detected by the study at slow time scale. Numerical results obtained from direct time integration of the system equations are compared with analytical predictions. At low energy, they show a qualitative agreement for the behavior of the chain of nonlinear oscillators and a quantitative one for the main structure. 
At higher energy, the numerical behavior of the overall system at slow time scale is precisely predicted by analytical time multi-scale developments. Besides, it has been proven that the response of the chain can be described by a nonlinear normal mode approach: during periodic regimes at relatively high energies, one mode is excited, while during strongly modulated regimes the behavior of the chain is the result of repeated bifurcations between nonlinear modes of the system. Then, the detection of the geometry of the slow invariant manifold and equilibrium and singular points is used to provide evidence of passive control of the linear oscillator. It is shown on a given example that energy of the main structure can be transferred in the chain of oscillators in order to mitigate its vibrations. Finally, a discussion on how the explained methodology can be used to study a multi-degree-of-freedom main linear system coupled to the same chain by projecting its dynamics on one of its modes of interest is given. These developments can be used for designing an appropriate nonlinear chain for localizing or focalizing the vibratory energy of targeted modes of main systems into the chain.

As stated above, the modal behaviors of the chain have been studied in this work, whereas the existence of localized waves and breathers has not been addressed. An abundance of works on such solutions in sole nonlinear chains (without coupling the main system) can be found in the literature. For example, the existence of breathers has been discussed by Cretegny et al. [40], Iooss and James [41], or by James et al. [42] in FermiPasta-Ulam chains with Hertz contact, and more generally in nonlinear lattices by Aubry [43]. Similarly, Starosvetsky and Manevitch [44] have studied localization and energy exchange in a periodic dimer FermiPasta-Ulam chain, while Perchikov and Gendelman [45] have discussed existence and stability of discrete breathers in a chain with vibro-impact potentials. However, in the above-mentioned studies, contrary to the present work, the dynamics of the chain alone is considered. It would then be interesting to see if spatially localized solutions such as breathers or solitons can exist in the present system where the coupling with the main system shall play an important role.

Acknowledgements The authors would like to thank the following organizations for supporting this research: (i) The "Ministère de l'Environnement, de l'Énergie et de la Mer" and (ii) LABEX CELYA (ANR-10-LABX-0060) of the "Université de Lyon" within the program "Investissement d'Avenir" (ANR-11-IDEX-0007) operated by the French National Research Agency (ANR).

\section{References}

1. Gendelman, O.V., Manevitch, L.I., Vakakis, A.F., M'Closkey, R.: Energy pumping in nonlinear mechanical oscillators: part I-dynamics of the underlying Hamiltonian systems. J. Appl. Mech. 68, 34-41 (2000)

2. Vakakis, A.F., Gendelman, O.V.: Energy pumping in nonlinear mechanical oscillators: part II—resonance capture. J. Appl. Mech. 68, 42-48 (2000)

3. Gendelman, O.V.: Transition of energy to a nonlinear localized mode in a highly asymmetric system of two oscillators. Nonlinear Dyn. 25, 237-253 (2001)

4. Vakakis, A.F., Gendelman, O.V., Bergman, L.A., McFarland, D.M., Kerschen, G., Lee, Y.S.: Nonlinear Targeted Energy Transfer in Mechanical and Structural Systems. Springer, Dordrecht (2009)

5. Zhu, S.J., Zheng, Y.F., Fu, Y.M.: Analysis of non-linear dynamics of a two-degree-of- freedom vibration system with nonlinear damping and non-linear spring. J. Sound Vib. 271, 15-24 (2004)

6. Gatti, G., Brennan, M.J.: On the effects of system parameters on the response of a harmonically excited system consisting of weakly coupled nonlinear and linear oscillators. J. Sound Vib. 330, 4538-4550 (2011)

7. Sapsis, T.P., Vakakis, A.F., Bergman, L.A.: Effect of stochasticity on targeted energy transfer from a linear medium to a strongly nonlinear attachment. Prob. Eng. Mech. 26, 119-133 (2011)

8. Luongo, A., Zulli, D.: Aeroelastic instability analysis of NES-controlled systems via a mixed multiple scale/harmonic balance method. J. Vib. Control 20, 1985-1998 (2014)

9. Charlemagne, S., Lamarque, C.H., Ture Savadkoohi, A.: Dynamics and energy exchanges between a linear oscillator and a nonlinear absorber with local and global potentials. J. Sound Vib. 376, 33-47 (2016)

10. Lee, Y.S., Vakakis, A.F., Bergman, L.A., McFarland, D.M., Kerschen, G.: Suppressing aeroelastic instability using broadband passive targeted energy transfers, part 1: theory. AIAA J. 45, 693-711 (2007)

11. Shao, J., Cochelin, B.: Theoretical and numerical study of targeted energy transfer inside an acoustic cavity by a non-linear membrane absorber. Int. J. Non-Linear Mech. 64, 85-92 (2014)

12. Gendelman, O.V.: Targeted energy transfer in systems with non-polynomial nonlinearity. J. Sound Vib. 315, $732-745$ (2008)

13. Gendelman, O.V.: Analytic treatment of a system with a vibro-impact nonlinear energy sink. J. Sound Vib. 331, 4599-4608 (2012)

14. Ture Savadkoohi, A., Lamarque, C.H.: Vibratory energy localization by non-smooth energy sink with time-varying mass. Appl. Non-Linear Dyn. Syst. 93, 429-442 (2014)

15. Lamarque, C.H., Ture Savadkoohi, A.: Dynamical behavior of a Bouc-Wen type oscillator coupled to a nonlinear energy sink. Meccanica 49, 1917-1928 (2014)

16. Weiss, M., Ture Savadkoohi, A., Gendelman, O.V., Lamarque, C.H.: Dynamical behavior of a mechanical system including Saint-Venant component coupled to a nonlinear energy sink. Int. J. Non-Linear Mech. 63, 10-18 (2014) 
17. McFarland, D., Kerschen, G., Kowtko, J.J., Lee, Y., Bergman, L., Vakakis, A.F.: Experimental investigation of targeted energy transfers in strongly and nonlinearly coupled oscillators. J. Acoust. Soc. Am. 118, 791-799 (2005)

18. Kerschen, G., McFarland, D., Kowtko, J.J., Lee, Y.S., Bergman, L., Vakakis, A.F.: Experimental demonstration of transient resonance capture in a system of two coupled oscillators with essential stiffness nonlinearity. J. Sound Vib. 299, 822-838 (2007)

19. Vakakis, A.F., Manevitch, L.I., Gendelman, O.V., Bergman, L.A.: Dynamics of linear discrete systems connected to local, essentially non-linear attachments. J. Sound Vib. 264, 559-577 (2003)

20. Vakakis, A.F., McFarland, D.M., Bergman, L.A., Manevitch, L.I., Gendelman, O.V.: Isolated resonance captures and resonance capture cascades leading to single- or multi-mode passive energy pumping in damped coupled oscillators. J. Vib. Acoust. 126, 235-244 (2004)

21. Starosvetsky, Y., Gendelman, O.: Interaction of nonlinear energy sink with a two degrees of freedom linear system: internal resonance. J. Sound Vib. 329, 1836-1852 (2010)

22. Gendelman, O.V., Manevitch, L.I.: Reflection of short rectangular pulses in the ideal string attached to strongly nonlinear oscillator. Chaos Solitons Fractals 11, 2473-2477 (2000)

23. Vakakis, A.F., Manevitch, L.I., Musienko, A.I., Kerschen, G., Bergman, L.A.: Transient dynamics of a dispersive elastic wave guide weakly coupled to an essentially nonlinear end attachment. Wave Motion 41, 109-132 (2005)

24. Manevitch, L.I., Gendelman, O.V., Musienko, A.I., Vakakis, A.F., Bergman, L.A.: Dynamic interaction of a semi-infinite linear chain of coupled oscillators with a strongly nonlinear end attachment. Phys. D 178, 1-18 (2003)

25. Rothos, V.M., Vakakis, A.F.: Dynamic interactions of traveling waves propagating in a linear chain with a local essentially nonlinear attachment. Wave Motion 46, 174-188 (2009)

26. Vaurigaud, B., Ture Savadkoohi, A., Lamarque, C.H.: Targeted energy transfer with parallel nonlinear energy sinks. Part I: design theory and numerical results. Nonlinear Dyn. 66, 763-780 (2011)

27. Ture Savadkoohi, A., Vaurigaud, B., Lamarque, C.H., Pernot, S.: Targeted energy transfer with parallel nonlinear energy sinks, part II: theory and experiments. Nonlinear Dyn. 67, 37-46 (2012)

28. Panagopoulos, P.N., Vakakis, A.F., Tsakirtzis, S.: Transient resonant interactions of finite linear chains with essentially nonlinear end attachments leading to passive energy pumping. Int. J. Solids Struct. 41, 6505-6528 (2004)

29. Tsakirtzis, S., Kerschen, G., Panagopoulos, P.N., Vakakis, A.F.: Multi-frequency nonlinear energy transfer from linear oscillators to MDOF essentially nonlinear attachments. J. Sound Vib. 285, 483-490 (2005)

30. Tsakirtzis, S., Panagopoulos, P.N., Kerschen, G., Gendelman, O., Vakakis, A.F., Bergman, L.A.: Complex dynamics and targeted energy transfer in linear oscillators coupled to multi-degree-of-freedom essentially nonlinear attachments. Nonlinear Dyn. 48, 285-318 (2007)

31. Wierschem, N., Luo, J., Al-Shudeifat, M., Hubbard, S., Ott, R., Fahnestock, L., Quinn, D., McFarland, D., Spencer Jr., B., Vakakis, A., Bergman, L.: Experimental testing and numerical simulation of a six-story structure incorporating two-degreeof-freedom nonlinear energy sink. J. Struct. Eng. 140, 04014027 (2014)

32. Starosvetsky, Y., Vakakis, A.F.: Primary wave transmission in systems of elastic rods with granular interfaces. Wave Motion 48, 568-585 (2011)

33. Ture Savadkoohi, A., Lamarque, C.H., Weiss, M., Vaurigaud, B., Charlemagne, S.: Analysis of the 1:1 resonant energy exchanges between coupled oscillators with rheologies. Nonlinear Dyn. (2016). doi:10.1007/s11071-016-2792-3

34. Nayfeh, A., Mook, D.: Nonlinear Oscillations. Wiley, Hoboken (1979)

35. Manevitch, L.I.: The description of localized normal modes in a chain of nonlinear coupled oscillators using complex variables. Nonlinear Dyn. 25, 95-109 (2001)

36. Starosvetsky, Y., Gendelman, O.V.: Strongly modulated response in forced 2DOF oscillatory system with essential mass and potential asymmetry. Phys. D 237, 1719-1733 (2008)

37. Rosenberg, R.M.: Normal modes of nonlinear dual-mode systems. J. Appl. Mech. 27, 263-268 (1960)

38. Rosenberg, R.M.: The normal modes of nonlinear n-degree-of-freedom systems. J. Appl. Mech. 29, 7-14 (1962)

39. Rosenberg, R.M.: On nonlinear vibrations of systems with many degrees of freedom. Adv. Appl. Mech. 9, 155-242 (1966)

40. Cretegny, T., Dauxois, T., Ruffo, S., Torcini, A.: Localization and equipartition of energy in the $\beta$-FPU chain: Chaotic breathers. Phys. D 121, 109-126 (1998)

41. Iooss, G., James, G.: Localized waves in nonlinear oscillator chains. Chaos 15, 015113 (2005)

42. James, G., Kevrekidis, P.G., Cuevas, J.: Breathers in oscillator chains with Hertzian interactions. Phys. D 251, 39-59 (2013)

43. Aubry, S.: Breathers in nonlinear lattices: existence, linear stability and quantization. Phys. D 103, 201-250 (1997)

44. Starosvetsky, Y., Manevitch, L.I.: On intense energy exchange and localization in periodic FPU dimer chains. Phys. D 264, 66-79 (2013)

45. Perchikov, N., Gendelman, O.V.: Dynamics and stability of a discrete breather in a harmonically excited chain with vibroimpact on-site potential. Phys. D 292-293, 8-28 (2015) 